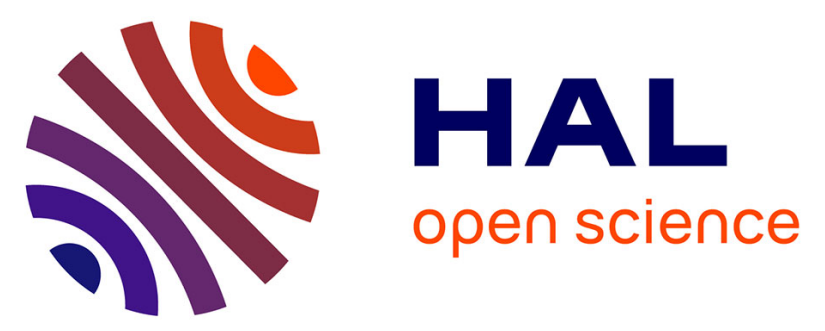

\title{
Color and aerosol changes in Jupiter after a North Temperate Belt disturbance
}

S. Perez-Hoyos, A. Sanchez-Lavega, J. F. Sanz-Requena, N. Barrado-Izagirre, O. Carrión-González, A. Anguiano-Arteaga, P. G. J. Irwin, Ashwin Braude

\section{- To cite this version:}

S. Perez-Hoyos, A. Sanchez-Lavega, J. F. Sanz-Requena, N. Barrado-Izagirre, O. Carrión-González, et al.. Color and aerosol changes in Jupiter after a North Temperate Belt disturbance. Icarus, 2020, 352 (December), pp.114031. 10.1016/j.icarus.2020.114031 . insu-02912559

HAL Id: insu-02912559

https://hal-insu.archives-ouvertes.fr/insu-02912559

Submitted on 30 Dec 2021

HAL is a multi-disciplinary open access archive for the deposit and dissemination of scientific research documents, whether they are published or not. The documents may come from teaching and research institutions in France or abroad, or from public or private research centers.
L'archive ouverte pluridisciplinaire HAL, est destinée au dépôt et à la diffusion de documents scientifiques de niveau recherche, publiés ou non, émanant des établissements d'enseignement et de recherche français ou étrangers, des laboratoires publics ou privés. 


\title{
Color and aerosol changes in Jupiter after a North Temperate Belt disturbance
}

\author{
S. Pérez-Hoyos ${ }^{1}$, A. Sánchez-Lavega ${ }^{1}$, J.F. Sanz-Requena ${ }^{2,3}$, N. \\ Barrado-Izagirre $^{1}$, O. Carrión-González ${ }^{4}$,A. Anguiano-Arteaga ${ }^{1}$, P.G.J. Irwin ${ }^{5}$, \\ A. S. Braude
${ }^{1}$ Dpto. Física Aplicada I, Faculty of Engineering Bilbao, Universidad del País Vasco UPV/EHU, 48013 Bilbao, Spain
${ }^{2}$ Dpto. Ciencias Experimentales, Universidad Europea Miguel de Cervantes, 47012 Valladolid, Spain
${ }_{3}^{3}$ Dpto. de Física Teórica, Atómica y Óptica, Universidad de Valladolid, 47011 Valladolid, Spain
${ }^{4}$ Zentrum für Astronomie und Astrophysik, Technische Universität Berlin, D-10623 Berlin, Germany
${ }^{5}$ Department of Physics, Atmospheric, Oceanic and Planetary Physics, University of Oxford, Oxford, UK
${ }^{6}$ Laboratoire Atmosphères, Milieux, Observations Spatiales (LATMOS), UVSQ Université Paris-Saclay, Sorbonne Université, CNRS, Paris, France

\section{Key Points:}

- We model the reflectivity change of Jupiter's North Temperate Belt between 2016 and 2017.

- A model with the chromophore well mixed within the tropospheric haze better reproduces the observed limb-darkening.

- The disturbance changed the particle concentration at upper tropospheric levels. 


\begin{abstract}
The banded appearance of Jupiter's atmosphere shows significant changes over time, sometimes even transforming the reflectivity of a whole latitudinal band in a few weeks, and staying for years with an aspect different from the usual one. The origin of some of these disturbances may be associated with the creation and destruction of the chromophore species that provides Jovian clouds their reddish coloration. In this work, we have focused on the North Temperate Belt (NTB) disturbance detected during the second flyby of Juno mission (NASA) on October 2016, as a series of convective storms interacted with the fastest zonal jet on Jupiter at $24^{\circ} \mathrm{N}$ over months and left a quiet belt characterized by an intense red coloration (Sánchez-Lavega et al., 2017). In order to determine the corresponding changes in the upper clouds and hazes we have used images taken in 2016 and 2017 with the Hubble Space Telescope Wide Field Camera 3. Such images were acquired before and after the outbreak, showing an intense color change in a narrow latitude band. The images cover the wavelength range from $250 \mathrm{~nm}$ up to the methane absorption band at $890 \mathrm{~nm}$, thus sensitive to a number of atmospheric levels from the lower stratosphere to the upper troposphere where the ammonia condensation cloud is expected to be located. Here we use the radiative transfer suite NEMESIS (Irwin et al., 2008) to determine the vertical distribution and properties of the upper hazes that best match the observed dependence of reflectivity with wavelength and geometry. We use two models for the Jovian chromophore: (A) an extended layer whose imaginary refractive index is left as a free parameter; and (B) a concentrated chromophore as in Sromovsky et al. (2017) using the optical properties by Carlson et al. (2016). Both scenarios show an increase in the number of particles responsible for the blue absorption approximately by a factor of 2 , and require only small changes in the rest of the atmospheric parameters. We find that, even though results provided by scenario B are also compatible with observations, the limb-darkening is better described by scenario A, where there is also an increase of the particle absorption at the shortest wavelengths. In this work, we also provide an extension of the expected imaginary refractive indices to wavelengths beyond those covered in previous laboratory works, which will be useful for future studies.
\end{abstract}

\title{
Plain Language Summary
}

The origin of color in Jupiter is one of the most persistent and intriguing questions in the study of Solar System atmospheres. Although Jupiter's coloration is very subtle to the human eye, there are several locations on the planet where the atmosphere looks redder than in other locations, which are white. The most famous of these locations is the Great Red Spot, a long-lived anticyclone in the Southern Hemisphere. To make things even more interesting, some places change their colors from time to time going from white to red, or vice versa, in a very short time (days or weeks). One such region is what we call the North Temperate Belt, which reddened at the end of 2016 and was observed by Hubble Space Telescope just before and after the process happened. We use these observations to test models of the red particles that give Jupiter its characteristic appearance and their vertical distribution. In particular, we test a recently proposed model of absorbing particles heavily concentrated in a very thin layer, the so-called "crème brûlée" model, against a model in which the particle absorption is distributed in an extended haze. Although both models are compatible with observations, we find that the latter reproduces the observed coloration better.

\section{Introduction}

The origin of color in Jupiter's atmosphere has been the subject of debate for decades (Owen \& Terrile, 1981; Ordonez-Etxeberria et al., 2016). The main components of Jovian upper clouds are white in chemical equilibrium: this is the case of the uppermost ammonia ice cloud, predicted to form in the upper troposphere of the planet. On the other hand, the observed variety of shades of brown and red require disequilibrium species to be explained 
(West et al., 2004). The origin and nature of the so-called "chromophore" is still unknown, although a relevant proposal has been made recently (Carlson et al., 2016). However, there are still some clues that might point to the presence of two or more chromophores present at the same time in Jupiter's atmosphere (Simon-Miller et al., 2001b; Strycker et al., 2011; Ordonez-Etxeberria et al., 2016).

The distribution of red coloration is not uniform in Jupiter and some vortices have attracted substantial attention in this respect (Sánchez-Lavega et al., 2013), the most wellknown case being the Great Red Spot (Baines et al., 2019). Some vortices are also known for temporal changes in color, typically from white to red (Pérez-Hoyos et al., 2009), without any observed or substantial modification of its height or dynamics (Hueso et al., 2009). There are also reported observations of red rings forming or disappearing in vortices (de Pater, Wong, et al., 2010). This opens a largely unexplored field of research on the relationship between dynamics, or more specifically vorticity, with the red chromophore.

In addition to this, some latitude bands of Jupiter are known to undergo periodic changes in their appearance. Leaving aside the North Temperate Belt that is discussed below, there is the case of the South Equatorial Belt fade (Pérez-Hoyos et al., 2012) during which a belt is transformed briefly into a zone-like region without any apparent cause or change in the observed dynamics.

There is a common understanding that the chromophore is located in the upper troposphere, at or above the expected ammonia condensation level (Simon-Miller et al., 2001a). West et al. (2004) reviewed the proposed candidates for the blue absorption up to the beginning of this century; however, none of them could be conclusively accepted or rejected by observations. Carlson et al. (2016) demonstrated that photolysed ammonia reacting with acetylene produces compounds with an absorption consistent with the values by Strycker et al. (2011) and others. The refractive indices of such compounds were successfully used to reproduce spectra acquired by Cassini/VIMS at various locations of the planet (Sromovsky et al., 2017) including the Great Red Spot (Baines et al., 2019). However, for this compound to reproduce the observations, the chromophore layer should be concentrated on top of the tropospheric haze and be relatively thin, in what has been called the "crème brûlée" model. One of the most important questions for this scenario is the production rate of acetylene for which thunderstorms were invoked as a possible explanation (Baines et al., 2019).

Convincing as the case for the "crème brûlée" model might be, Braude et al. (2020) found it difficult to reproduce the observed limb-darkening of red features in the Jovian atmosphere with this model, as fits by Sromovsky et al. (2017) were only given at few locations on the disk. Braude et al. (2020) also argue that it is possible to find a universal chromophore, at least within observation and model uncertainties, but their results provide a steeper blue-absorption gradient than that using the Carlson et al. (2016) chromophore.

The motivation of this work is that limb-darkening is a valuable tool for addressing this problem, rather than, or in addition to, spectral resolution. However, studying limbdarkening for individual features, frequently very active and variable, is not an easy task. For this reason we identified the North Temperate Belt of Jupiter as an ideal case of study of the limb-darkening behaviour for the suggested chromophores.

The North Temperate Belt (NTB) of Jupiter is centered around the fastest jet of the planet, at $24^{\circ} \mathrm{N}$, but its latitudinal boundaries are very variable. During the time at which these observations were made, the low albedo region was located approximately between $22^{\circ} \mathrm{N}$ and $28^{\circ} \mathrm{N}$ (Sánchez-Lavega et al., 2017). The disturbances of the North Temperate Belt (NTBDs) are known to happen roughly every four or eight Earth years (Rogers \& Adamoli, 2019), the last two events taking place in 2007 (Sánchez-Lavega et al., 2008) and 2016. The last one happened shortly before the second Juno spacecraft perijove (PJ2) which allowed detailed observations of its evolution (Sánchez-Lavega et al., 2017) that permitted dynamical simulations performed after the first event (García-Melendo et al., 2005) to be 


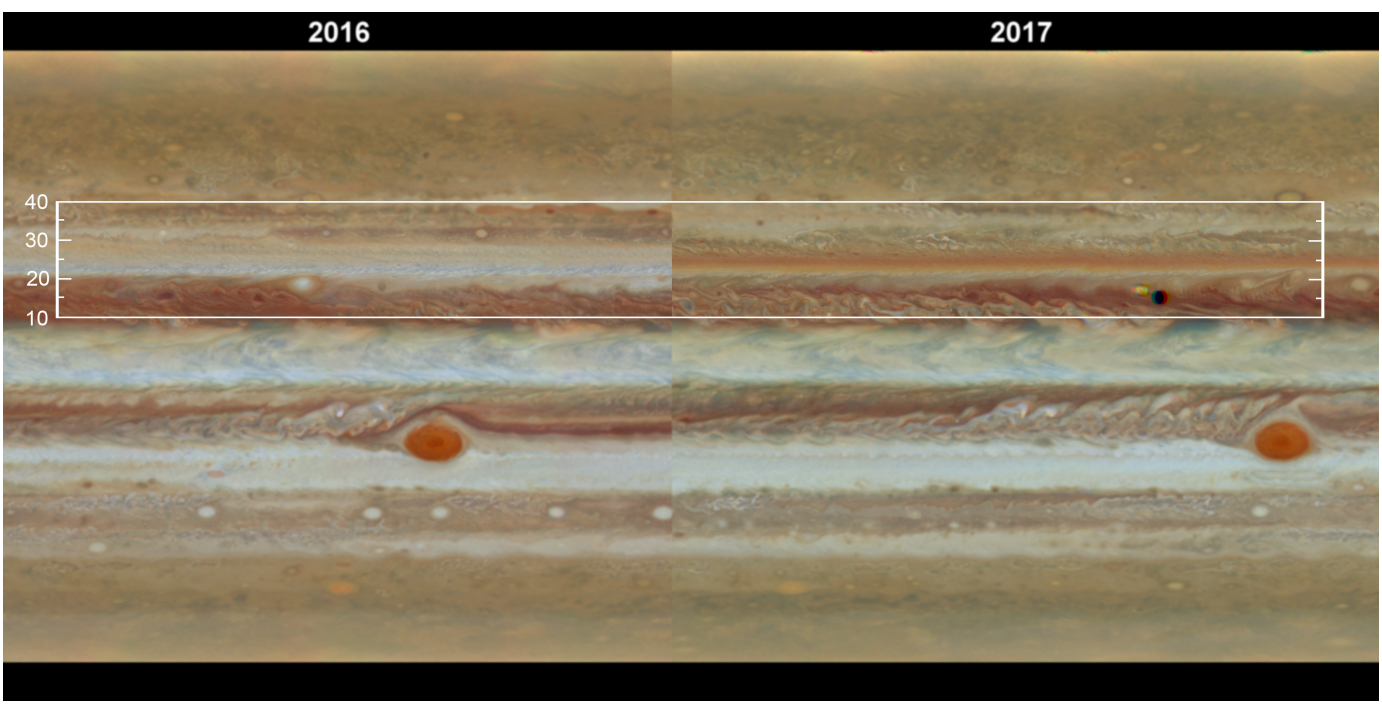

Figure 1. Color compositions of the HST OPAL images used in this work. The white box indicates the latitudes of interest where the changes after the North Temperate Belt disturbance are most apparent, including planetocentric latitudes

- These colour maps maps can be downloaded from the Barbara A. Mikulski Archive for Space Telescopes (https://archive.stsci.edu/prepds/opal/).

refined. Details can be found in the aforementioned references but, in short, all NTBDs start with an outbreak of one to three independent plumes leading to a planetary-scale disturbance. Once the turbulence originated by the 2016 plumes settled down, the entire latitudinal band transforms from being white into a reddish and homogeneous band (see Fig. 1). This is very appealing for our purposes, as the limb-darkening is much easier to determine in homogeneous latitude bands, providing an excellent framework for testing chromophore models.

It must be noted that changes in the reflectivity of the NTB might be accompanied (Rogers, 2009) by variations of lesser amplitude in neighboring latitudes, in particular the North North Temperate Belt (NNTB) at northern latitudes, and the North Equatorial Belt (NEB) closer to the Equator. Those variations have been scarcely reported and seldom analyzed in detail, to the best of our knowledge. Moreover, they tend to be not zonally homogeneous so they will not be considered in this work.

The goal of this work is to use observations from the Hubble Space Telescope (HST) of the North Temperate Belt just before and after the 2016 disturbance to determine the limb-darkening behaviour at visible and near-ultraviolet and near-infrared wavelengths. The dependence of the observed reflectivity from a spectral and geometrical point of view will be used to constrain the physical properties of atmospheric aerosols by means of a radiative transfer model. In particular, we will explore a scenario following the "crème brûlée" model (Sromovsky et al., 2017; Baines et al., 2019), fixing particle absorption to the values provided by Carlson et al. (2016), with the chromophore concentrated in a physically thin layer in the upper troposphere. We will also explore a second scenario in which the chromophore is well-mixed within the tropospheric haze and leaving the effective imaginary refractive index of the mixture (which affects the single scattering albedo) as a free parameter.

The structure of this paper is as follows. We describe the HST observations in Section 2, then in Section 3 we will show the retrieved limb-darkening and color indices of the images following the calibration in absolute photometry. The observations will be modeled with the 
Table 1. Summary of HST observations used in this work

\begin{tabular}{llcc}
\hline Date & \multicolumn{1}{c}{ Filters } & Number of images & Program \\
\hline 2016-02-09/10 & F275W, F343N, F395N, F467M, & 117 & OPAL \\
& F502N, F547M, F631N, FQ889N & & \\
2017-04-03 & F275W, F343N, F395N, F467M, & 105 & OPAL \\
& F502N, F547M, F631N, FQ889N & & \\
\hline
\end{tabular}

NEMESIS radiative transfer suite (Irwin et al., 2008) in Section 4 under the two scenarios described above. Finally, in Section 5 we will summarize the main conclusions of this work regarding the nature and distribution of the Jovian chromophore.

\section{HST Observations}

For this work we have used archived images taken by Hubble Space Telescope using the Wide Field Camera 3 (WFC3) instrument (Dressel, 2019). These images were obtained under the Outer Planet Atmospheres Legacy (OPAL) program (Simon et al., 2015). A summary of the observations used in this work can be found in Table 1 and an example of the images taken by this program is shown in Fig. 1. The 2016 NTBD event started in October 2016 and reached a planetary scale by December 2016; therefore, we cover the pre-outburst and planetary-scale stages of the disturbance.

The HST/WFC3 imaging covers near-ultraviolet to near-infrared wavelengths and includes the deep methane band filter at $889 \mathrm{~nm}$ (FQ889N). Filters are also well-selected to account for the aerosol absorption (Simon et al., 2015), in particular covering the blue range where the chromophore absorption is the strongest (West et al., 2004). Such or similar filter selection has proven in the past to be sensitive to the distribution of particles in the upper Jovian troposphere (Pérez-Hoyos et al., 2012). We show in Figure 2 the distribution of HST/WFC3 filters (Dressel, 2019) with respect to the geometric albedo of the planet and how they match the most interesting features of the planetary spectrum in this wavelength range.

A discussion on contribution functions for similar HST/WFC3 filters can be found in de Pater, Fletcher, et al. (2010). In short, UV filters are Rayleigh dominated but have substantial contributions from the sub-micron sized aerosols at the upper tens of mbar of the atmosphere. FQ889N filter, on the other hand, is methane-dominated and most influenced by the aerosols in the upper troposphere (around 100-200 mbar). The intermediate filters are all aerosol-dominated and could reach very deep levels in a cloud-free atmosphere. In a real, cloudy Jovian atmosphere, those filters will have contributions from the upper thick cloud, regardless of its location at the ammonia or $\mathrm{NH}_{4} \mathrm{SH}$ condensation level. This is particularly true for filter F631N with the lowest Rayleigh contribution. However, an exact description of the contribution function is model-dependent and varies with the particle size and vertical distribution.

All images used here have been calibrated in absolute reflectivity following the description given in Dressel (2019). Radiance values will be given in units of reflectivity $I / F$, or the ratio of the observed intensity to that of a perfect Lambertian reflector illuminated at normal incidence, as for example in Pérez-Hoyos et al. (2012).

In this work, we are interested in the zonal average of the reflectivity, not taking into account local variations due to the presence of particular atmospheric features such as vortices. In particular, we will focus in a region where the reflectivity is dominated by the 


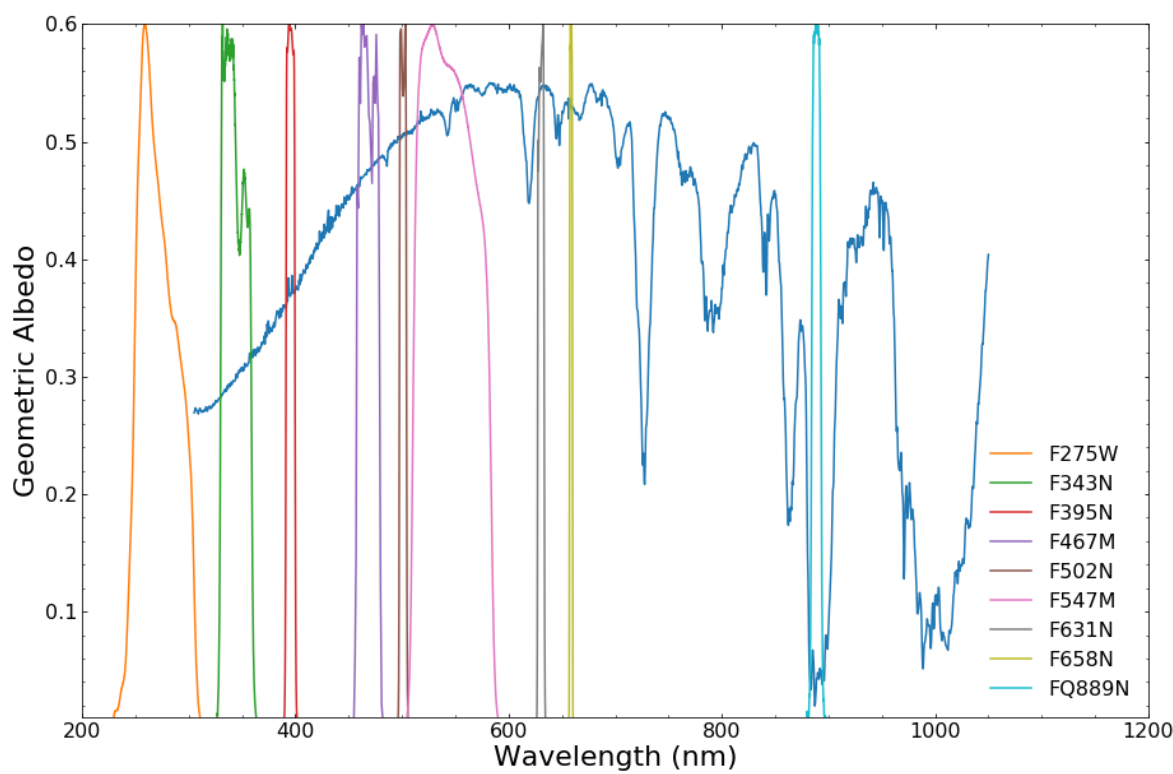

Figure 2. HST/WFC3 filters used in this work together with the geometric albedo spectrum of Jupiter obtained by Karkoschka (1998). Transmissivity of the filters are scaled to the same maximum only for representation purposes.

zonal average, with differences in the east to west direction only due to the limb-darkening. For this reason, we have used all the images taken in a given filter at the same time. For every latitude, we analyze all the images at the same time as will be explained in section 3.1. This is a way to smooth over individual features while preserving the zonal information contained in the images.

\section{Analysis}

\subsection{Limb-darkening analysis}

The dependence of the reflectivity on the incidence and emission angles can be reproduced with notable accuracy through an empirical law first introduced by Minnaert (1941). The process for applying this law to correct observations from limb-darkening can be found, for example, in Barrado-Izagirre et al. (2009). Here we are interested in retrieving the limb-darkening information for every latitude and filter. The Minnaert law can be written as:

$$
\frac{I}{F}=\left(\frac{I}{F}\right)_{0} \cdot \mu_{0}^{k} \cdot \mu^{k-1}
$$

Where $I / F$ is the observed reflectivity, $(I / F)_{0}$ is the nadir-viewing reflectivity, $k$ is the limb-darkening parameter. All these parameters depend on wavelength, but this dependency has been removed from equation 1 for simplicity. $\mu$ and $\mu_{0}$ are, respectively, the cosines of the emission and incidence angles. Such angles can be computed from the position of the Sun and the observer for any given time and location on the planetary disk (Horak, 1950). It is very convenient to express the Minnaert law in a linear way, by taking logarithms: 


$$
\ln \left(\mu \frac{I}{F}\right)=\ln \left(\frac{I}{F}\right)_{0}+k \cdot \ln \left(\mu \mu_{0}\right)
$$

Using Equation 2 it is possible to retrieve Minnaert parameters $(I / F)_{0}$ and $k$ by performing a least-squares fit of $\ln (\mu I / F)$ vs $\ln \left(\mu \mu_{0}\right)$. We show the results of this fitting as a function of latitude in Figure 3. The error bars in the Minnaert parameters come from the local variations at a given latitude, producing dispersion of observed reflectivity, but they can also be attributed to navigation uncertainties, in particular the errors associated with the limb-darkening parameter $k$. These uncertainties will be very important at a later stage, as they will propagate into the actual error bars that will be used during the radiative transfer analysis.

As can be seen in Figure 3, changes in the appearance of the NTB affect both the nadirviewing reflectivity $(I / F)_{0}$ and the limb-darkening parameter $k$. They are most apparent at shorter wavelengths, particularly at F343N and F395N, getting noticeably smaller as we move to longer wavelengths, in particular at filter F658N. Most affected latitudes are those around $20^{\circ} \mathrm{N}$, although there are some variations of smaller intensity up to $35^{\circ} \mathrm{N}$. The disturbance affected the meridional wind profile from $22^{\circ} \mathrm{N}$ to $28^{\circ} \mathrm{N}$ (Sánchez-Lavega et al., 2017). $I / F$ changes at other latitudes, related to the NNTB and the NEB, might also be important and related to the meridional transport of the disturbance (Rogers, 2009). It is worth mentioning that changes in the blue and near-UV filters are accompanied by a substantial change also in the deep methane band filter FQ889N, which gets brighter in similar latitudes as where the red absorption increases. This gives preliminary information on how the density of the chromophore increased after the disturbance.

These results can be viewed in terms of the spectral dependence of the Minnaert parameters, as is shown in Figure 4. Even for the latitudes of most variation, the changes are localized at blue wavelengths, and are the highest for filter F467M. It should be noted that the scale does not allow a fair comparison with filter FQ889N where the relative changes can be large even though absolute reflectivity values are small. Those changes are best seen when plotting the Minnaert parameters as a function of latitude, as in Figure 3. This gives an indication of the spectral change in reflectivity undergone by the Jovian atmosphere during the disturbance. These spectra, or similar ones, will be later analyzed in section 4 by means of a radiative transfer model in order to reproduce the observed reflectivity and the limb-darkening at the same time.

\subsection{Color indices}

Sánchez-Lavega et al. (2013) provided a simple way to estimate the color and altitude of clouds in the Jovian atmosphere by definining two indices. The first one is the altitudeopacity index (AOI), computed as the ratio between the methane band reflectivity and that observed in the near ultraviolet. High values of this index imply clouds with substantial opacity at higher levels in the atmosphere, as they would be bright in the methane band (due to the lack of methane absorption) and dark in the UV (due to the lack of Rayleigh scattering). On the other hand, the color index (CI) is a measure of the chromophore spectral slope, computed as the ratio of the reflectivity at blue and red wavelengths. Red features score low values (as they are dark in the blue and bright in the red wavelengths), while white or bluish features obtain the highest values. These values can be normalized to a reference region such as the South Tropical Zone (STrZ), which looks very homogeneous and white in all images (Sánchez-Lavega et al., 2013). The definition of the filters used for each index is loose, as it depends on the availability and the source of data; therefore, variations on the exact values of indices from one work to another might be expected.

Although this scheme has been used in other publications, Ordonez-Etxeberria et al. (2016) extended these indices not only to individual features but also to the overall banded aspect of the planet, as seen by Cassini ISS. Even the distribution of the indices within 

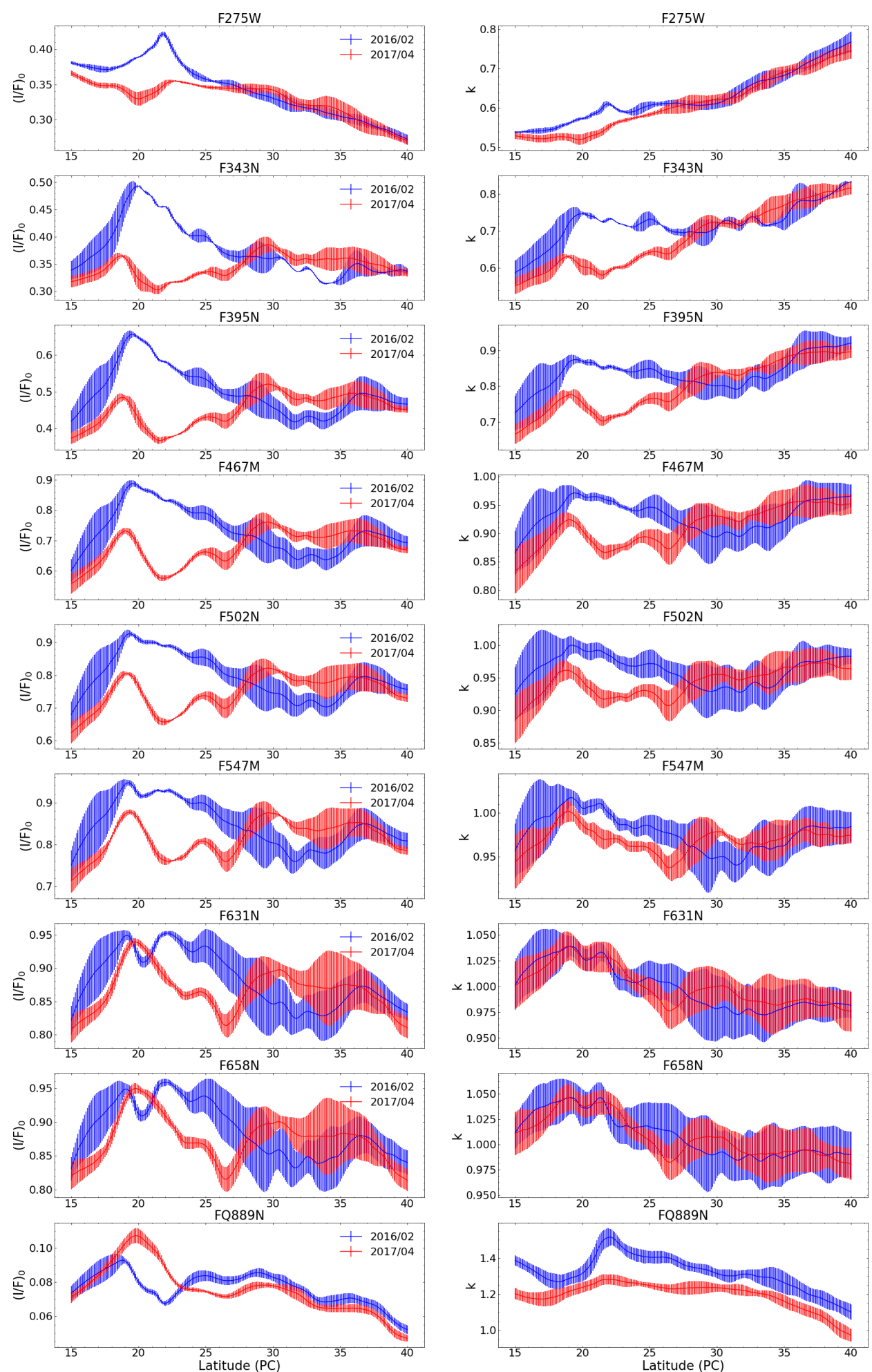

Figure 3. Minnaert parameters as a function of planetocentric latitude (PC) for dates shown in Table 1 in all available filters. Nadir viewing reflectivity $(I / F)_{0}$ is displayed in the left-hand column and limb darkening coefficient $k$ in the right. Blue lines are used for measurements prior to the NTB disturbance and red lines for data after the event. Please note the blended error bars. 

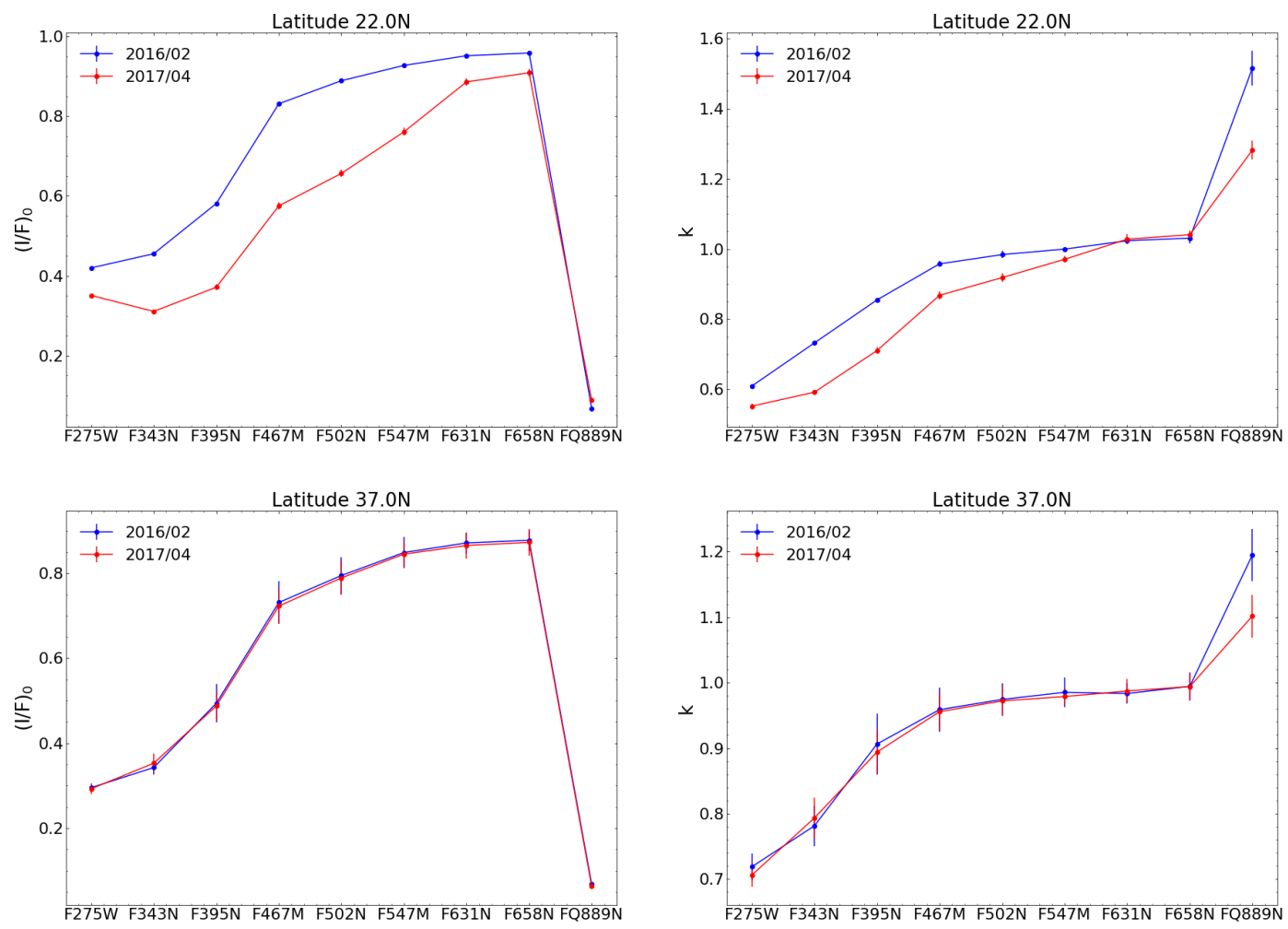

Figure 4. Spectral variation of Minnaert parameters for latitudes of maximum $\left(22^{\circ} \mathrm{N}\right)$ and minimum $\left(37^{\circ} \mathrm{N}\right)$ variation. Left-hand column displays the nadir viewing reflectivity $(I / F)_{0}$, while the right-hand column shows the limb darkening coefficient $k$. 


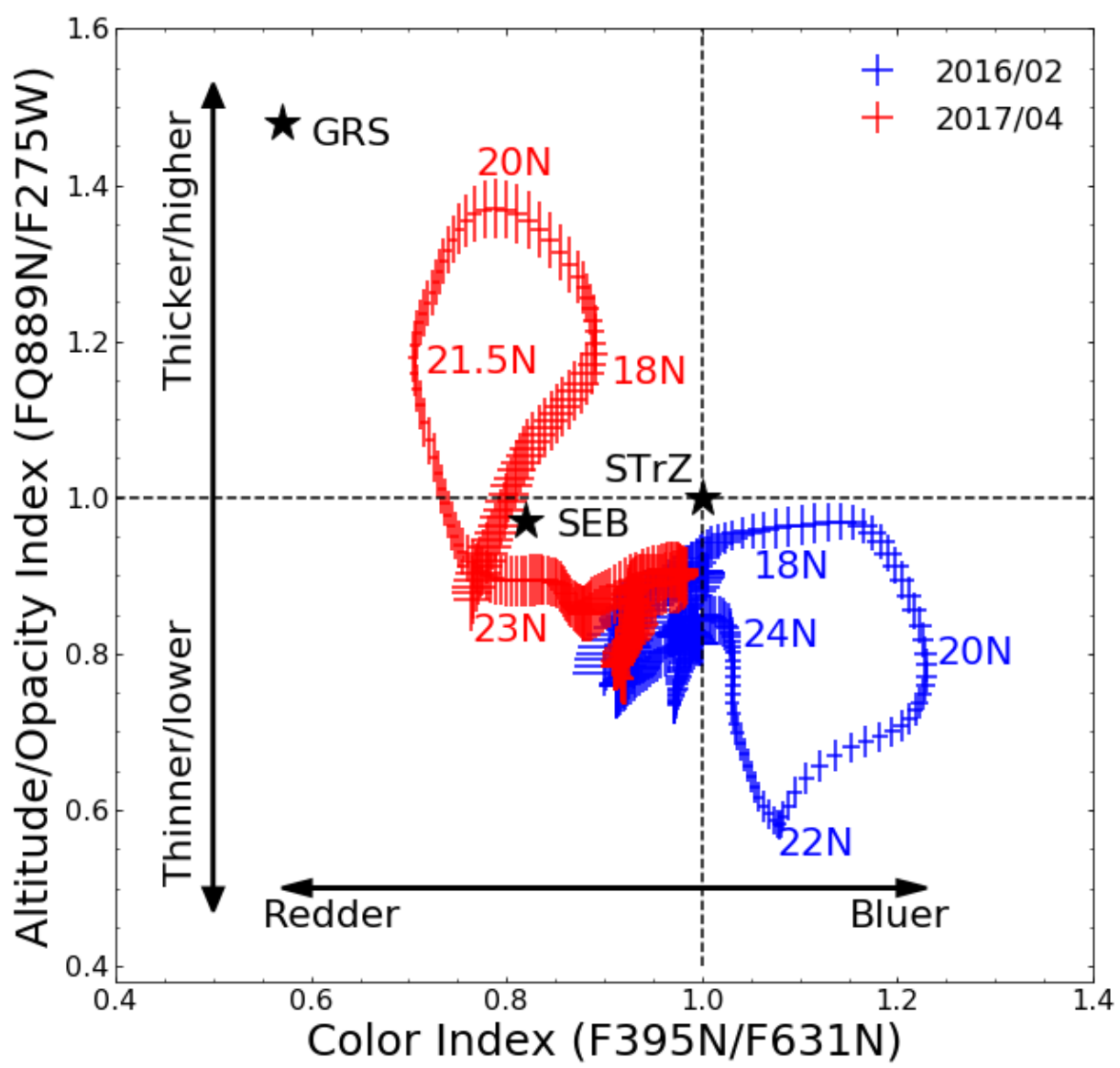

Figure 5. Altitude/Opacity index versus Color index for the latitudes of interest in 2016 (blue) and 2017 (red). Values are given as zonal averages with the error bars showing the 1- $\sigma$ dispersion of the indices. Latitudes with extreme values of the indices are shown for clarity. The STrZ is used for reference $(\mathrm{CI}=1.0, \mathrm{AOI}=1.0)$, values for the center of the GRS and the SEB are taken from Sánchez-Lavega et al. (2013)

the individual features (such as the Great Red Spot or oval BA) can be analyzed if images provide enough spatial resolution.

The computation of these simple indices from the OPAL images for 2016 and 2017 is shown in Figure 5. We show in the figure the latitudes for extreme values of the indices, but please note that they increase continuously with latitude, so the loops should be followed clockwise in 2016 and counter-clockwise in 2017. In our case, we compute the altitude-opacity index as the ratio between FQ889N and F275W data (AOI $=$ FQ889N/F275W), while the color index is given as the ratio between F395N and F631N (CI = F395N/F631N). It must be noted that calibrated data were used here without any Minnaert correction, latitudinal values were computed as zonal averages for longitudes within $20^{\circ}$ from the central meridian, to avoid limb-darkening issues. In fact, this can be extended closer to the limbs (up to $40^{\circ}$ from the central meridian, at least) without significant deviations. 
This analysis provides a useful insight into what happened in the region after the disturbance. The region between $15^{\circ} \mathrm{N}$ and $40^{\circ} \mathrm{N}$ is essentially redder and lower in altitude than the reference zone in the STrZ. While in 2016 the mean values of the indices are $\mathrm{CI}(2016)=1.00 \pm 0.08, \mathrm{AOI}(2016)=0.82 \pm 0.08$, the latitude band was noticeably redder, as images suggest, and somewhat higher or thicker, as $\mathrm{CI}(2017)=0.87 \pm 0.08, \mathrm{AOI}(2017)=0.9 \pm$ 0.2 . However, not all latitudes contribute the same to the change and there are two loops in the figure that illustrate the changes happening between $17^{\circ} \mathrm{N}$ and $25^{\circ} \mathrm{N}$. In particular, the region between $20^{\circ} \mathrm{N}$ and $22^{\circ} \mathrm{N}$ goes from the lower right of the graph to the upper left, i.e., from the bluest and lowest/thinnest clouds to display the reddest and highest/thickest clouds, not that far from the values of the core of the Great Red Spot (Sánchez-Lavega et al., 2013). If we exclude these changing latitudes, we go from $\mathrm{CI}(2016)=0.96 \pm 0.03$, $\mathrm{AOI}(2016)=0.82 \pm 0.04$ to $\mathrm{CI}(2017)=0.92 \pm 0.03, \mathrm{AOI}(2017)=0.85 \pm 0.04$. This means slightly redder clouds with similar altitudes in 2017 compared with 2016. Relative to other Jovian belts or zones, they are lower and redder than the South Tropical Zone (STrZ), used here for reference, but lower and bluer than the South Equatorial Belt (SEB). Please note that the values of the GRS or the SEB may vary in time and are only given for the times reported in Sánchez-Lavega et al. (2013).

The use of these indices is a quick approach to the state of the atmosphere, which in this case indicates latitudinal diffusion of chemical species formed at high altitudes or alternatively ascending from deeper levels in the central latitude around $22^{\circ} \mathrm{N}$. Although some other minor changes can be identified, these are the most important variations in the altitude and color of the particles. Hopefully, a more detailed quantitative analysis of the atmospheric parameters, such as the one presented in the following section, should provide a quantitative description compatible with the discussion presented above.

\section{Radiative Transfer Analysis}

Given the Minnaert parameters shown in section 3.1, it is possible to reconstruct the average reflectivity under any viewing and illumination geometry. For example, the spectra in the left column of Figure 4 correspond to a perfect nadir-viewing geometry (i.e. $\mu=\mu_{0}=1.0$ ). As we want to ensure that our retrievals also reproduce the observed limbdarkening, we used seven synthetic spectra for each latitude, obtained from the aforementioned Minnaert parameters, at different distances from the central meridian (i.e., the point closer to the nadir-viewing geommetry) or zenithal angles. We arbitrarily chose to sample points separated by $10^{\circ}$ in longitude in order to constrain the limb-darkening behaviour of the model pretty well. The sampling can be seen in Figure 6 or Figure 8. Each spectral point has an uncertainty associated with the uncertainties in the Minnaert parameters described in previous sections. The error bars in the Minnaert parameters result in an average 10\% contribution to the total uncertainty in each reflectivity value, which was assumed as the observational uncertainty for our modeling purposes. These values are including not just the reflectivity errors, but also navigation uncertainties and spatial variations in reflectivity due to relatively small variations in vertical structure. Systematic uncertainties were discarded, as direct comparison of North to South scans along central meridian for both years showed an excellent agreement and any systematics, if present, would affect both years similarly.

\subsection{Model atmosphere}

The NEMESIS radiative transfer suite (Irwin et al., 2008) was used for the retrieval of the physical parameters of the atmosphere. NEMESIS utilizes an optimal estimator approach (Rodgers, 2000) to find the most likely value of the parameters defining the atmospheric model, starting from an a priori description of the atmosphere and the observational uncertainties. NEMESIS is able to find the atmospheric parameters that best reproduce the observed reflectivity as a function of wavelength for all geometries simultaneously, as well as their associated uncertainties, depending on how sensitive the model is to each one. As such, 
it is not always easy to discern between two competing scenarios, other than favoring the one with lower deviation from the observed data provided that both models have the same number of free parameters. In this work, we will study two situations where the vertical distribution of aerosols is assumed to be organized in a quite different way.

The description of the gaseous component of the atmosphere is common for the two aerosol scenarios. The composition of the atmospheric gas was taken from Taylor et al. (2004). Gravitational acceleration is computed from the coefficients tabulated in Astronomical Almanac (1994) and latitudinal dependence was considered. The Rayleigh scattering is computed for a mixture of $\mathrm{H}_{2}$ and $\mathrm{He}$, while the only gases with noticeable absorption bands for the filters used here are $\mathrm{CH}_{4}$ and $\mathrm{NH}_{3}$. These absorptions are stored in pre-computed k-tables which can be easily accessed during retrievals. For doing so we used the absorption coefficients by Karkoschka and Tomasko (2010) for methane, and those by Coles et al. (2018) for ammonia. The temperature profile has little or no impact even in the strongest methane absorption band and was assumed to be that of Seiff et al. (1998). We did not take into account Raman scattering by Hydrogen, as its effect is below 0.03 in absolute reflectivity at $300 \mathrm{~nm}$ for the aerosol-dominated atmosphere of Jupiter as shown in Karkoschka (1994). Details on how NEMESIS works can be found in Irwin et al. (2008) - here we used a correlated-k, doubling-adding version of the code for a plane-parallel atmosphere.

We show in tables 2 and 3 the parameters describing the particle component in the two scenarios used here, with two particular solutions at the same latitude $\left(22^{\circ} \mathrm{N}\right)$ for the two years. Parameters can be fixed if their value is assumed to be known or which cannot be constrained using current data, free if they are left as free parameters, or computed if they are not directly used by the model but obtained from some other parameters that can be free or fixed. This is the case for the optical thickness, which is not actually used by the model but computed from the particle density distribution and optical properties. Unless stated otherwise, all wavelength dependent properties are given at $890 \mathrm{~nm}$. Finally, it must be noted that the a priori values are described by a starting value and an a priori uncertainty. Such uncertainty does not mean that only values within the initial range are tested, as NEMESIS is able to explore values outside it by a number of times the initial uncertainty. However, small a priori relative errors will focus the search closer to the initial value than huge a priori relative errors. A full explanation of this method based on the optimal estimator scheme can be found, for example, in Rodgers (2000) or in Hanel et al. (1992).

All particle ensembles are modeled using a Mie phase function with the corresponding size distribution parameters given in tables 2 and 3. However, as Jovian particles are not expected to be spherical (West et al., 2004; Zhang et al., 2013),their phase functions are smoothed over using fits to double Henyey-Greenstein functions, thus removing features as rainbows or glories which have not been observed on Jupiter. In any case, phase functions are only relevant for comparisons with previous works, as here we are modeling observations taken at similarly low phase angles, almost at the backscattering lobe of the phase function.

The first aerosol scenario A is one with an extended chromophore, as described in Table 2. Although we considered using a model similar to that by Braude et al. (2020) with a continuous profile of conservative scatterers together with a gaussian distribution of the chromophore, we found that this left too many free parameters for our spectral resolution.

The scenario A model used here is very similar to what we have used in previous investigations, both for Jupiter (Pérez-Hoyos et al., 2009, 2012) and Saturn (Pérez-Hoyos et al., 2016; Sanz-Requena et al., 2018, 2019). In this model, we have two haze layers above a bottom cloud, putatively formed by ammonia ice (West et al., 2004). Here, the upper stratospheric haze will extend from $P_{1}=1$ to $P_{2}=100$ mbar (Pérez-Hoyos et al., 2009 ) with a constant number of particles per gram of atmosphere, thus representing the overall integrated thickness of the stratospheric aerosol $\tau_{s t r}$, as we assume no sensitivity to its vertical distribution. The a priori value of $\tau_{\text {str }}$ is also in agreement with the values retrieved 
Table 2. Scenario A: extended chromophore - parameters

\begin{tabular}{|c|c|c|c|c|c|}
\hline Layer & Parameter & Type & Value & $201622^{\circ} \mathrm{N}$ & $201722^{\circ} \mathrm{N}$ \\
\hline \multirow[t]{6}{*}{ Stratospheric haze } & $\mathrm{P}_{1}$ & Fixed & 1 mbar & & \\
\hline & $\mathrm{P}_{2}$ & Fixed & 100 mbar & & \\
\hline & $\tau_{\text {str }}$ & Free & $0.01 \pm 0.002$ & $0.001 \pm 0.01$ & $0.002 \pm 0.001$ \\
\hline & $\mathrm{m}_{r}, \mathrm{~m}_{i}$ & Fixed & $1.43,10^{-3}$ & & \\
\hline & $\mathrm{r}_{e f f}$ & Fixed & $0.15 \mu \mathrm{m}$ & & \\
\hline & $\sigma_{e f f}$ & Fixed & 0.1 & & \\
\hline \multirow[t]{7}{*}{ Tropospheric haze } & $\mathrm{P}_{b o t}$ & Free & $200 \pm 100$ mbar & $180 \pm 30$ mbar & $190 \pm 40$ mbar \\
\hline & $\mathrm{N}$ & Free & $100 \pm 50 \mathrm{part} / \mathrm{cm}^{3}$ & $190 \pm 30 \mathrm{part} / \mathrm{cm}^{3}$ & $280 \pm 70$ part $/ \mathrm{cm}^{3}$ \\
\hline & $\mathrm{H}$ & Free & $5 \pm 2 \mathrm{~km}$ & $1 \pm 6 \mathrm{~km}$ & $1 \pm 3 \mathrm{~km}$ \\
\hline & $\tau_{t r p}$ & Computed & $10 \pm 5$ & $10 \pm 2$ & $18 \pm 1$ \\
\hline & $\mathrm{m}_{r}, \mathrm{~m}_{i}$ & Free & Carlson et al. (2016) & & \\
\hline & $\mathrm{r}_{e f f}$ & Free & $1 \pm 1 \mu \mathrm{m}$ & $1.06 \pm 0.07 \mu \mathrm{m}$ & $0.79 \pm 0.08 \mu \mathrm{m}$ \\
\hline & $\sigma_{e f f}$ & Free & $0.1 \pm 0.1$ & $0.039 \pm 0.009$ & $0.05 \pm 0.02$ \\
\hline \multirow[t]{6}{*}{ Bottom cloud } & $\mathrm{P}_{5}$ & Fixed & 700 mbar & & \\
\hline & $\mathrm{P}_{6}$ & Fixed & 900 mbar & & \\
\hline & $\tau_{c l d}$ & Free & $100 \pm 10$ & $90 \pm 40$ & $80 \pm 50$ \\
\hline & $\mathrm{m}_{r}, \mathrm{~m}_{i}$ & Fixed & $1.43,10^{-8}$ & & \\
\hline & $\mathrm{r}_{e f f}$ & Fixed & $5 \mu \mathrm{m}$ & & \\
\hline & $\sigma_{e f f}$ & Fixed & 0.1 & & \\
\hline
\end{tabular}

by Zhang et al. (2013). These small particles (Pérez-Hoyos et al., 2012) are assumed to be effectively non-absorbing with arbitrarily fixed values of the refractive indices $m_{r}$ and $m_{i}$, although this assumption has no impact in the results. We also use fixed values of the effective radius $r_{\text {eff }}$ and variance of the log-normal distribution $\sigma_{e f f}$. Both the imaginary refractive indices and the particle size distribution values had already been used in PérezHoyos et al. (2012).

In scenario A the chromophore is well mixed within the upper tropospheric haze and we use a single particle description for the mixture of both. Hence, we will retrieve average values for the particle absorption (i.e., imaginary refractive index $m_{i}$ ) which would represent not the pure chromophore itself but its mixing with fresh material (possibly white ammonia ice) in the haze. The main drawback of this approach is that it is unable to separate the refractive indices of the chromophore from the primary condensate, as it will appear always mixed to a greater or lesser extent. In any case, we use as the a priori guess for the refractive indices $m_{r}, m_{i}$ the values obtained by Carlson et al. (2016) for their chromophore candidate, with an a priori uncertainty of $100 \%$ and extended with a constant value for wavelengths outside the original range. For the vertical distribution of this layer, it is possible to fit most limb-darkening observations in this wavelength range with a simplified scheme with only three free parameters for the tropospheric particle distribution. In this case, these will be the bottom pressure $P_{b o t}$, the scale height $H$ and the maximum peak abundance $N$, as stated in Table 2. While the total optical thickness for this layer is expected to be that from previous works Pérez-Hoyos et al. (2009, 2012), the other parameters are more difficult to determine from the preceding literature. $P_{b o t}$ and $H$ were selected to include the upper and lower levels in (Pérez-Hoyos et al., 2012). Finally, the a priori guess for the particle number density was scaled to retrieve sensible values for $\tau_{\text {trop }}$.

Below the hazes, a cloud is expected at the ammonia condensation level or below (e.g., as in West et al. (2004)). At these wavelengths, the atmosphere is already optically thick and our sensitivity is very low even in the best case. Here we assume a very crude model 
Table 3. Scenario B: crême-brûlée model - parameters

\begin{tabular}{lccccc}
\hline Layer & Parameter & Type & Value & $201622^{\circ} \mathrm{N}$ & $201722^{\circ} \mathrm{N}$ \\
\hline Stratospheric haze & $\mathrm{P}_{\text {str }}$ & Free & $40 \pm 40 \mathrm{mbar}$ & $5 \pm 5 \mathrm{mbar}$ & $5 \pm 5 \mathrm{mbar}$ \\
& $\tau_{s t r}$ & Free & $0.2 \pm 0.01$ & $0.01 \pm 0.01$ & $0.01 \pm 0.02$ \\
& $\mathrm{~m}_{r}, \mathrm{~m}_{i}$ & Fixed & $1.43,10^{-5}$ & & \\
& $\mathrm{r}_{\text {eff }}$ & Free & $0.1 \pm 0.1 \mu \mathrm{m}$ & $0.04 \pm 0.03 \mu \mathrm{m}$ & $0.05 \pm 0.04 \mu \mathrm{m}$ \\
& $\sigma_{\text {eff }}$ & Fixed & 0.1 & & \\
Chromophore layer & $\mathrm{P}_{c b}$ & Free & $200 \pm 200 \mathrm{mbar}$ & $200 \pm 20 \mathrm{mbar}$ & $130 \pm 20 \mathrm{mbar}$ \\
& $\tau_{c b}$ & Free & $0.2 \pm 0.2$ & $0.013 \pm 0.007$ & $0.04 \pm 0.02$ \\
& $\mathrm{~m}_{r}, \mathrm{~m}_{i}$ & Fixed & Carlson et al. $(2016)$ & & \\
& & Free & $(<400 \mathrm{~nm} ;>750 \mathrm{~nm})$ & & \\
& $\mathrm{r}_{\text {eff }}$ & Free & $0.2 \pm 0.1 \mu \mathrm{m}$ & $0.08 \pm 0.03 \mu \mathrm{m}$ & $0.08 \pm 0.03 \mu \mathrm{m}$ \\
& $\sigma_{\text {eff }}$ & Fixed & 0.1 & & \\
& $\mathrm{P}_{\text {cld }}$ & Free & $1.0 \pm 0.5 \mathrm{bar}$ & $0.8 \pm 0.8 \mathrm{bar}$ & $0.8 \pm 0.8 \mathrm{bar}$ \\
& $\tau_{c l d}$ & Free & $20 \pm 10$ & $28 \pm 6$ & $25 \pm 6$ \\
& $\mathrm{~m}_{r}, \mathrm{~m}_{i}$ & Fixed & Martonchik et al. $(1984)$ & & \\
& $\mathrm{r}_{\text {eff }}$ & Free & $1.0 \pm 0.5 \mu \mathrm{m}$ & $1.4 \pm 0.2 \mu \mathrm{m}$ & $1.3 \pm 0.2 \mu \mathrm{m}$ \\
& $\sigma_{\text {eff }}$ & Fixed & 0.1 & & \\
\hline
\end{tabular}

of a bottom cloud since there is little or no influence in the retrievals. It extends from $P_{5}$ to $P_{6}$ with constant density, refractive indices and particle size distribution. The properties proposed in Table 2 as a priori guesses are taken from Pérez-Hoyos et al. (2009, 2012).

The second scenario B is inherited from Sromovsky et al. (2017) and Baines et al. (2019). This model atmosphere was adapted to NEMESIS in (Braude, 2019) and (Braude et al., 2020), where further details can be found. The main difference with scenario A is that we find here the chromophore layer concentrated on top of the tropospheric haze (or cloud) in what has been called a crême-brûlée model. The physical parameters used for the a priori guess are shown in Table 3 and come from the aforementioned investigations. The main strength of this approach is that a physically thin and optically limited chromophore layer allows the determination of refractive indices of a pure chromophore layer. Hence, the use of a candidate, such as the one proposed by Carlson et al. (2016) allows testing for its spectral and geometrical response. It must be noted that those indices start at $400 \mathrm{~nm}$; for wavelengths shorter than that we left their value as a free parameter, starting from the extreme value reported by Carlson et al. (2016).

Here we find again a stratospheric haze at the highest levels, at pressure $P_{s t r}$, although in this case it is assumed to be physically thin. Below that, we find another thin layer composed of pure chromophore, in principle that by Carlson et al. (2016). For wavelengths outside the range studied by that work, we extrapolated a constant value equal to the extreme values of the refractive indices. The main parameters governing this layer are its pressure level $P_{C B}$ and opacity $\tau_{C B}$, as well as particle size $r_{e f f}$. This chromophore layer is located on top of the last aerosol layer, expected to be composed of ammonia ice or other fresh, non-absorbing material. This layer extends from $P_{c l d}$ and it will be responsible for most of the scattering at continuous wavelengths and it is similar to what we termed tropospheric haze in scenario A.

There are a number of obvious similarities and differences between both scenarios. The question here is how well each one is able to reproduce the spectral and geometrical behavior of the reflectivity, as it will be covered in the next section. 


\subsection{Results}

\subsubsection{Scenario A results}

We show in Figure 6 a summary of the results fitting the observations of 2016 and 2017 with scenario A, described in Table 2. Figure 6 shows that all fits are well below the marginal value $\chi^{2} / N=1.0$, thus convincingly fitting observations within their error bars. $\chi^{2} / N$ is computed as the average mean quadratic deviation between observations and bestfitting models, $N$ being the number of points, including wavelengths and geometries, fitted with each model, as for example described in Pérez-Hoyos et al. (2012). The reader should note that $\chi^{2} / N$ is one-dimensional approach to a multi-dimensional problem to quantify the goodness of fit and must be taken with care. As such an average value is not enough to describe the goodness of fit since it may hide deviations at certain wavelengths or geometries; we show in the same Figure 6 how an average model at a given latitude and time is able to reproduce first the spectral and then the geometrical (i.e., limb-darkening) dependence of the reflectivity. This aspect will be analyzed later in greater detail. Although there are some systematic deviations at the red continuum wavelengths (particularly at the F658N filter, potentially related to the deepest thick cloud as discussed in a previous section), it is possible to reproduce the overall observations satisfactorily with this scenario.

Most of the differences between latitudes are attributed in this case to the intermediate tropospheric haze, while the average properties of the upper stratospheric haze (basically a very optically thin aerosol layer) and the lower bottom cloud (essentially semi-infinite) remain constant in latitude. In the case of the upper layer, the optical thickness of the stratospheric haze increases with latitude, but it is always very low $\left(\tau_{s t r} \leq 0.002\right)$ for both years, with very similar values. For the lowest layer, the model favours a thick cloud deck $\left(\tau_{c l d} \geq 10\right)$, although with greater uncertainty.

As shown in Figure 7, the optical thickness of the tropospheric haze at the $890 \mathrm{~nm}$ reference wavelength is fairly constant in latitude for $2017\left(<\tau_{t r p}>=15 \pm 1\right)$ while there is a substantial decrease in the optical thickness in 2016 in the region between $20^{\circ} \mathrm{N}$ and $25^{\circ} \mathrm{N}$. In this band the aerosol opacity increases by a factor of two from $\sim 9$ to $\sim 18$, and this seems to be a key factor in explaining the reflectivity changes.

With respect to other parameters of the tropospheric haze, the relative error is particularly high for the bottom pressure of the haze, which shows no sensitivity and is found to be located at 200 mbar, as in the a priori guess, thus providing no information on its location. The scale height of the aerosols is better constrained and shows a clear dependence with latitude, increasing toward northern latitudes from a mere $2 \mathrm{~km}$ extent closer to the equator up to $7 \mathrm{~km}$ close to $40^{\circ} \mathrm{N}$, but with substantial error bars. Within such uncertainties, the differences in scale height from 2016 to 2017, which reach values of $20 \%$ in the region around $20^{\circ} \mathrm{N}$, certainly may play a role in the observed reflectivity variations.

When we come to analyze the particle mean size and its size distribution, shown in the middle panels of Figure 7, differences are clearer from 2016 and 2017, frequently beyond the retrieved error bars. In the case of the radius, it is around $0.92 \pm 0.05 \mu \mathrm{m}$ for both years, but differences in the problematic region goes from an increase of the particle mean radius of more than $30 \%$ at $22^{\circ} \mathrm{N}$ to a decrease of $15 \%$ around $20^{\circ} \mathrm{N}$. The width of the size distribution is very similar in 2016 and in 2017 , with slight changes between $20^{\circ} \mathrm{N}$ and $25^{\circ} \mathrm{N}$.

However, the particle absorption plays a more significant role in explaining the observed differences before and after the disturbance. We show the changes in the imaginary refractive index at two selected wavelengths in the bottom panels of Figure 7 . The absorption increases strongly, by up to a factor of two, in the latitudes between $20^{\circ} \mathrm{N}$ and $25^{\circ} \mathrm{N}$. As we model the refractive indices in $50 \mathrm{~nm}$ steps for computational purposes, we can see that changes start at $350 \mathrm{~nm}$ and end at $550 \mathrm{~nm}$, being negligible below and above those wavelengths. The strongest variations happen at $350 \mathrm{~nm}$ and $400 \mathrm{~nm}$. At these wavelengths, changes are clearly above the 1- $\sigma$ level. The optimization procedure also reduces substantially the 

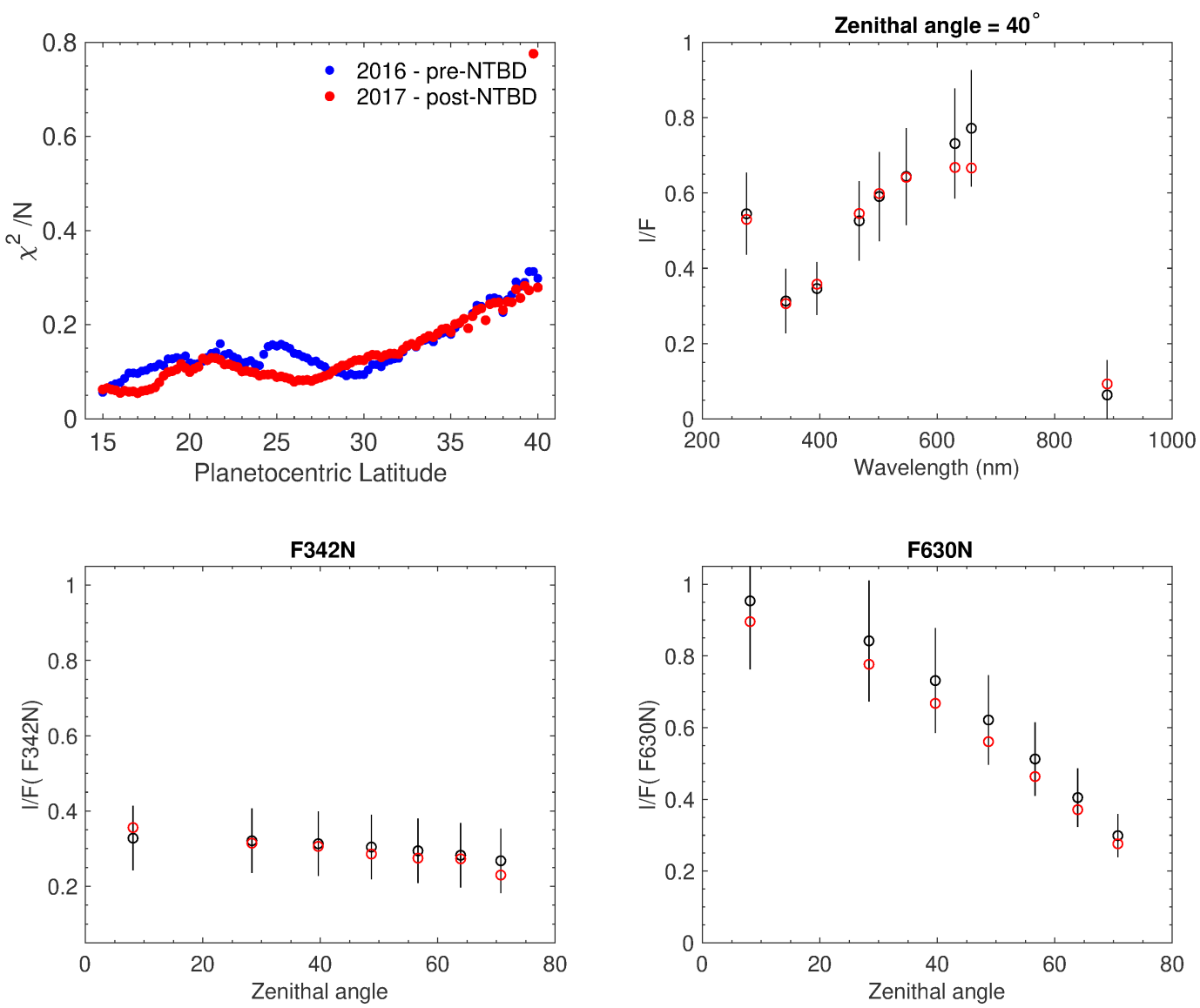

Figure 6. This figure summarizes the best-fitting results for scenario A. In the upper left plot, we show mean quadratic deviation of the best-ftting models for scenario A as a function of latitude for 2016 and 2017 observations. The rest of panels are devoted to the same specific case: $22^{\circ} \mathrm{N}$ in 2017 , the location of the most intense reflectivity change. Black circles are used here for observations and red circles for model results. Upper right panel represents the wavelength dependence of reflectivity for a given zenithal angle, while the bottom panels show the dependence of reflectivity with zenithal angle for two given filters: F342N (left, with almost no limb-darkening) and F630N (right, with strong limb darkening). 

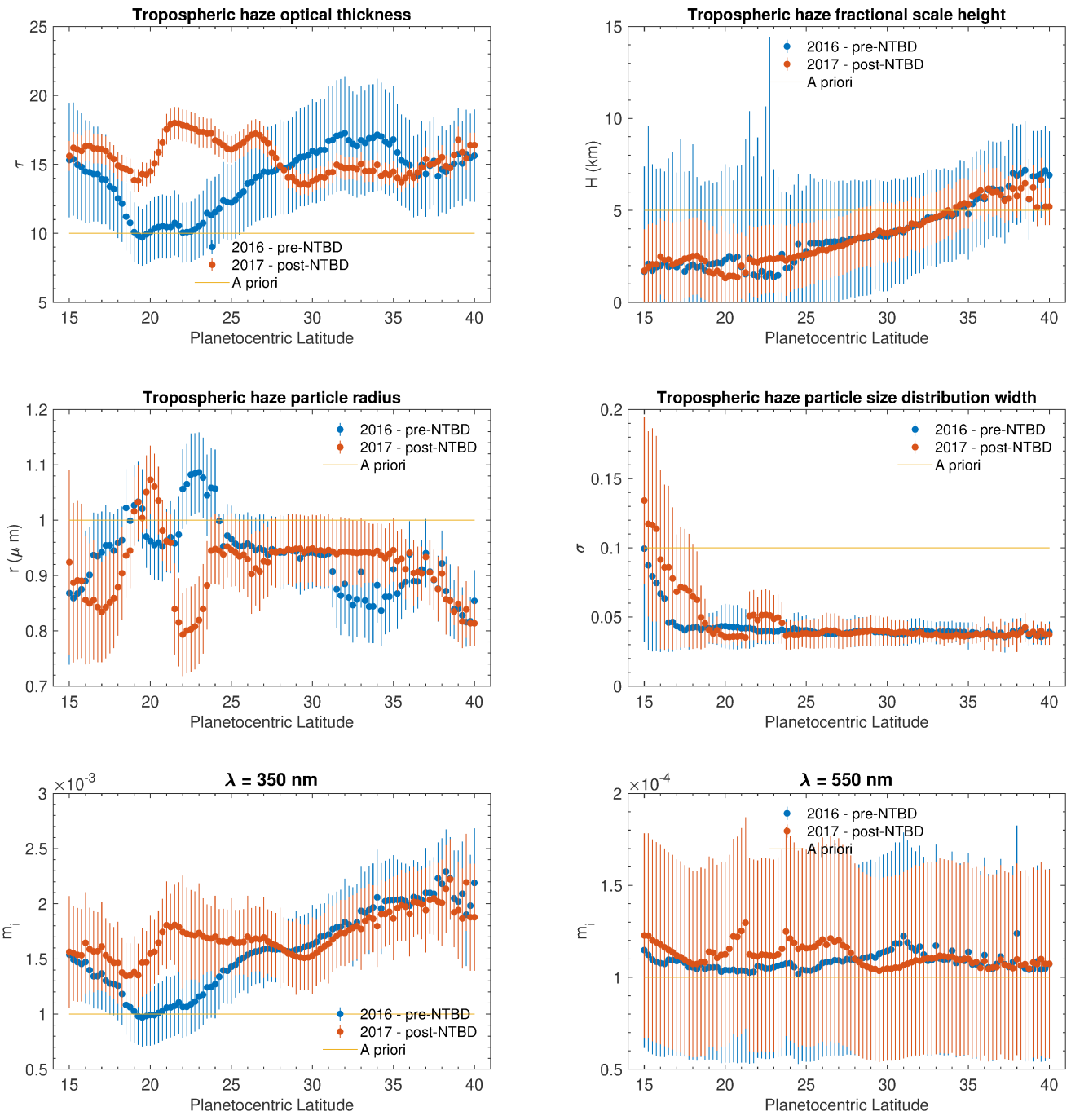

Figure 7. Best-fitting values defining the vertical distribution of the tropospheric haze for the most informing parameters in scenario A. Blue dots correspond to the values before the NTB disturbance (2016) and red dots after the disturbance (2017), while a priori guesses are in orange (see Table 2 for a priori uncertainties). In the upper row we shown the integrated optical thickness (left) and the particle scale height (right). The intermediate row is for the mean particle radius (left) and the width of the size distribution (right). The bottom row shows the values of the imaginary refractive index at $350 \mathrm{~nm}$ (left) and $550 \mathrm{~nm}$ (right). 

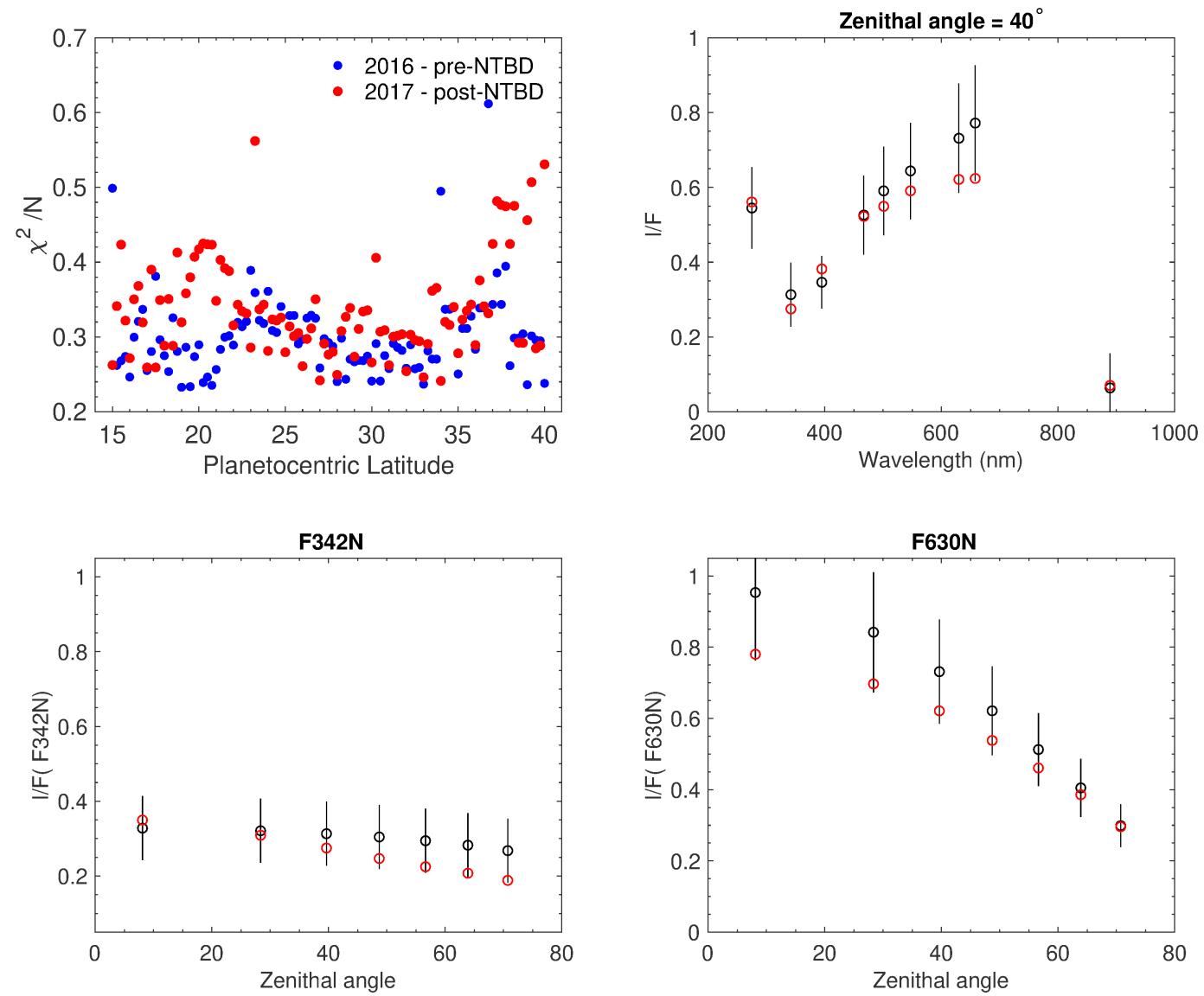

Figure 8. Same as Figure 6 but for scenario B.

relative error of the imaginary refractive index, from the a priori $100 \%$ relative uncertainty to an average of $30 \%$ at $350 \mathrm{~nm}$.

As we will discuss later, this change in the particle absorption within the limitations of the first scenario A cannot be attributed to a change of the absorbing species but, most likely, to a change in the concentration of the absorbing particles within the tropospheric haze or to the thickness of a plausible coating layer around non-absorbing droplets or ice particles.

\subsubsection{Scenario $B$ results}

Figure 8 shows that the mean quadratic deviation between observations and models following scenario $\mathrm{B}$ is substantially higher than in scenario A, being $\chi^{2} / N \sim 0.3$. The main problem is shown in the bottom panels of Figure 8, where it is clear that the limb-darkening obtained with this model is not accurate for most filters. While the overall spectral behaviour depicted in Figure 8 is correct, there is a systematic deviation at continuum wavelengths that does not allow our model to reach as good fits as in the preceding scenario.

In this scenario $\mathrm{B}$, the uppermost layer is composed of extremely small particles (around $0.05 \mu \mathrm{m})$ with a very low average optical thickness in the near infrared $\left(\tau_{S H}(890 \mathrm{~nm}) \sim\right.$ 0.01 ). It must be noted that such small particles provide a much higher value of the optical thickness in the ultraviolet, being $\tau_{S H}(275 n m) \geq 1.0$ in the shortest filters used in this work. The bottom pressure of this haze decreases with latitude, being located at around 100 mbar 

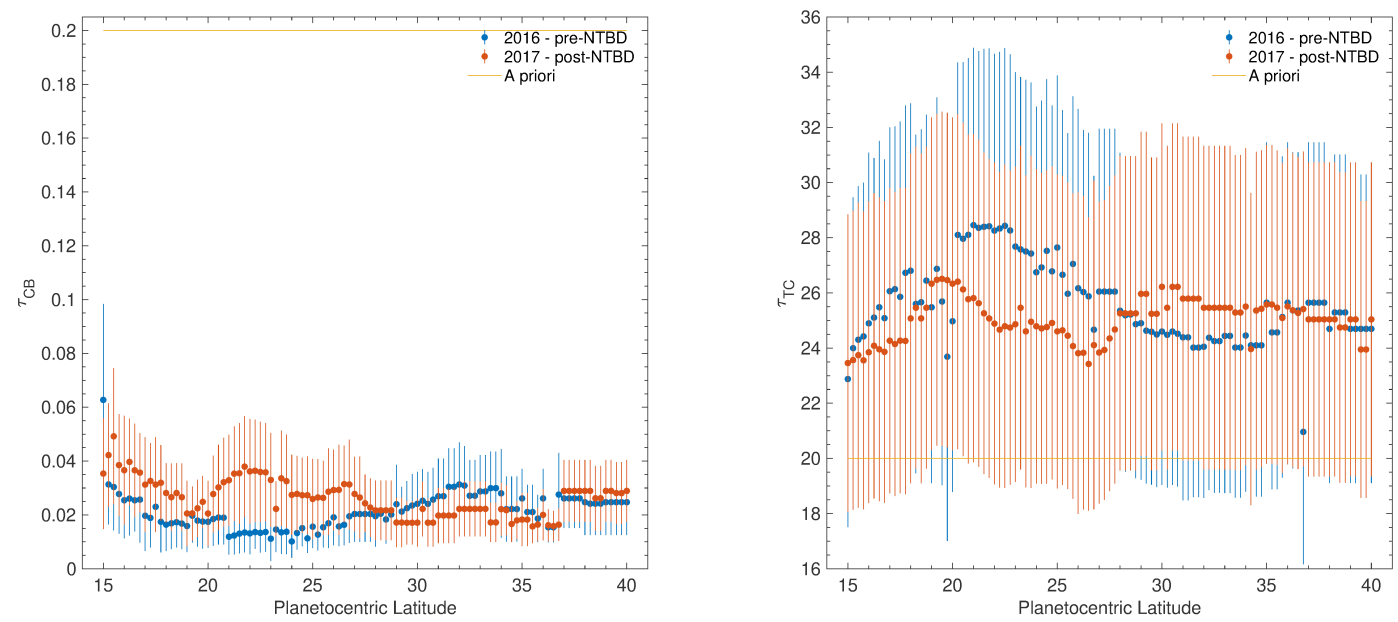

Figure 9. Main changes in the parameters of scenario B. (left) Optical thickness of the chromophore layer. (right) Optical thickness of the bottom cloud. Both are referred to the value at 890 nm.

at $15^{\circ} \mathrm{N}$ and less than 1 mbar at $40^{\circ} \mathrm{N}$. In any case, properties of this haze are very stable in time and are not required to explain the variations in the North Temperate Belt.

In this context, the "crème brûlée" or thin chromophore layer plays a more substantial role, as shown in Figure 9. Always located at $140 \pm 20 \mathrm{mbar}$, it is composed of small particles $\left(r_{C B} \sim 0.1 \mu \mathrm{m}\right)$ with a total optical thickness similar to those discussed for the stratospheric haze ( $\tau_{C B}$ from 1.25 to 0.03 , depending on wavelength). Both the belt-to-zone variations and the appearance changes after the NTB disturbance can be greatly controlled by tuning the optical thickness of this layer, with changes that can amount up to $100 \%$ in some latitudes within the most variable region from $20^{\circ} \mathrm{N}$ to $25^{\circ} \mathrm{N}$.

Finally, the bottom cloud also plays a role in scenario B (see Figure 9). A cloud with its base located around 1 bar and with a scale height similar to that of the gas works well for most situations. The particle radius is also quite stable $\left(r_{T C} \sim 1.35 \mu \mathrm{m}\right.$ and displays only small variations. The integrated optical thickness, however, changes by around 10-20\% and, even though within error bars, such changes seem to be correlated with the variations observed in reflectivity and focused again in similar latitudes as discussed in the preceding paragraph. While there is a possible problem of parameter degeneration at the longest wavelengths, as an increase of one parameter can be partially compensated by a decrease in the other, this has a limited effect due to the strong absorption in the blue and UV, as such an absorption is not present in the tropospheric cloud. Thus, the parameter coupling is broken by the simultaneous fitting of the whole spectrum.

However, there is yet another degree of freedom for the scenario B. As there is no information on the chromophore absorption at the shortest wavelengths (values given in Carlson et al. (2016) start at $400 \mathrm{~nm}$ ), we used a conservative a priori assumption with a constant value of $m_{i}$ shortwards of $400 \mathrm{~nm}$. Shortwards of that, the imaginary refractive values in scenario B were left as free parameters and fitted. However, the results are consistent in showing a maximum absorption around $300 \mathrm{~nm}$ and a decrease towards both sides of the spectrum. Note that, although within error bars, this differs from scenario A at the UV end of the absorption spectrum, as the extended chromophore model did not show a clear maximum in the particle absorption. The slope at longer wavelengths was forced to that by Carlson et al. (2016). On the other hand, the absorption at shortest wavelengths is very similar for all latitudes and both years, before and after the disturbance. We also show in 


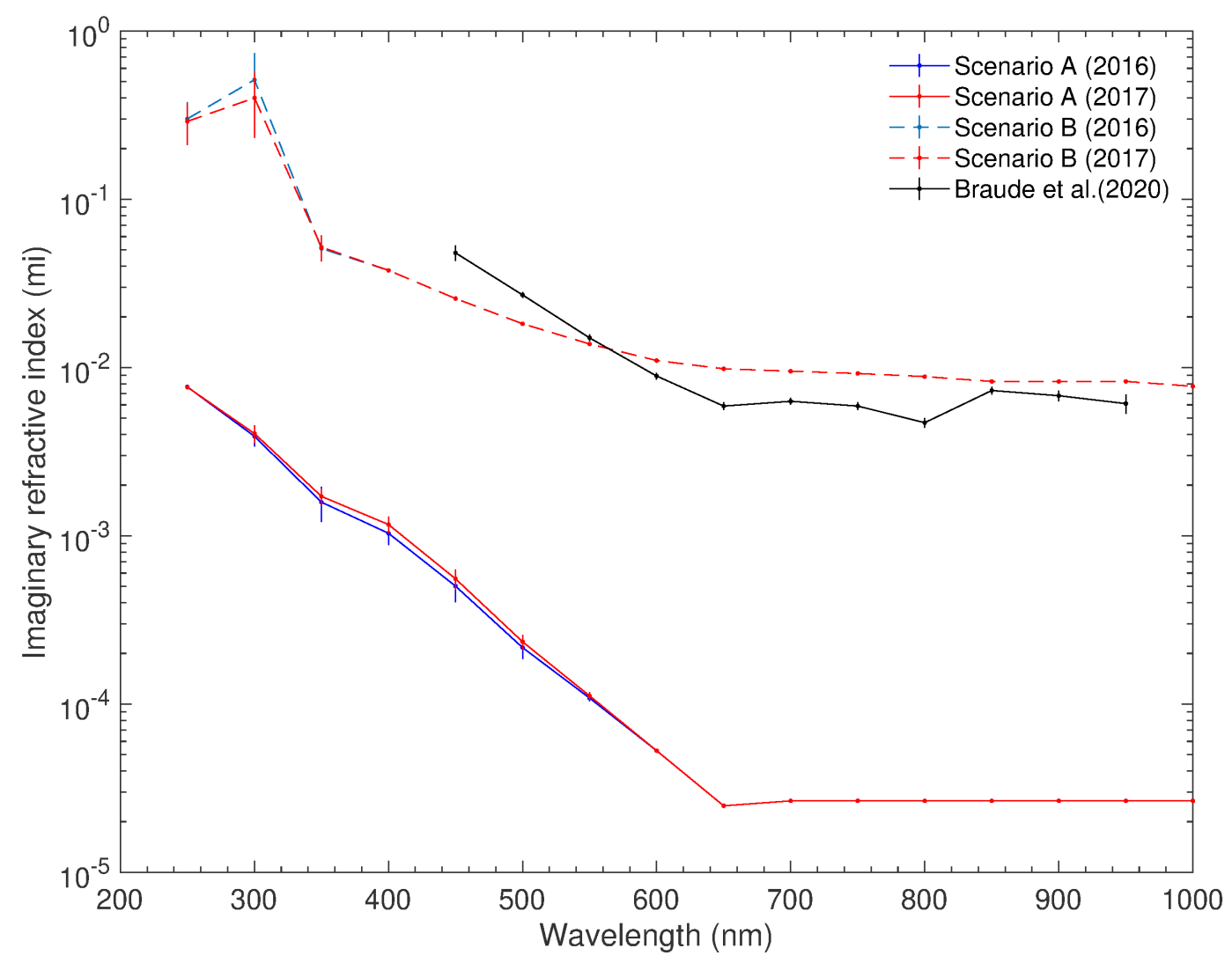

Figure 10. Average absorption of the tropospheric particles (scenario A, solid lines) or the chromophore layer (scenario B, dashed lines) for 2016 (blue) and 2017 (red). The results by Braude et al. (2020) are shown in black for comparison. Note that the scenario B values are those by Carlson et al. (2016), while values were fitted outside the range covered in that study. Scenario A values were fitted at all wavelengths.

Figure 10, the values retrieved by Braude et al. (2020), with an approach to the vertical distribution of the chromophore somewhat different to that used in this work or in others.

\subsection{Discussion}

We have inspected two different scenarios and evaluated their ability to fit the observed limb-darkening for a region of Jupiter that changes dramatically in a very short time span. We showed in Figure 11 the performance of both scenarios in fitting the observed limbdarkening at three selected filters: one near the blue (where the change is the strongest), one in the red (with minimum variations) and the filter at the $889 \mathrm{~nm}$ deep methane band (which is essential to determine the vertical distribution of tropospheric particles). Even though scenario A performs better in general terms, scenario B is better in reproducing the limb-darkening observed in the deep methane band filter FQ889N in 2017, while both fit 2016 observations at that filter similarly well. Figure 11 demonstrates that there is room for improvement in both scenarios, as neither reproduces the observed limb-darkening in all situations. Note that we are fitting the spectra individually and then comparing the retrieved limb-darkenings with the observed ones. This means that we are fitting the points and not the trend, so small variations from one side to the other can result in larger deviations from the observed and modelled trend. In future works, limb-darkening could be 

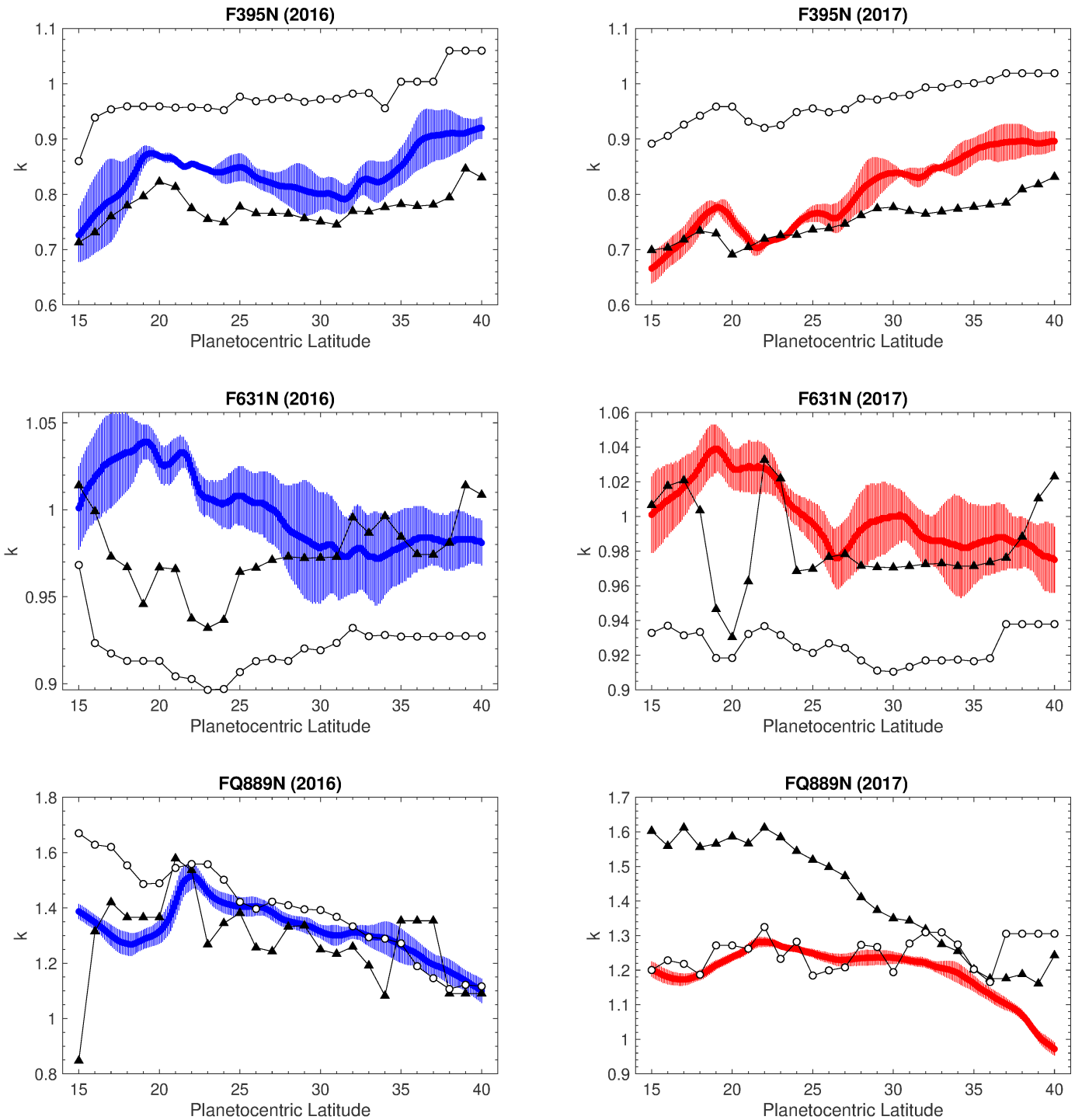

Figure 11. Limb-darkening $k$ coefficient from equation 1 computed for scenarios A and B. 2016 observations are shown in left column, in blue, and 2017 values on the right, red colour. For scenario A limb-darkening values we use black triangles and white circles for values obtained from scenario B. Values are shown every $1^{\circ}$ for clarity.

used as an additional constraint during the fitting procedure and not just as a validation of the process.

In scenario A the changes in reflectivity in the NTB are caused by an increase of the optical thickness of the tropospheric haze together with a substantial increase in the particle absorption at the blue wavelengths. As we have already commented, this can be attributed to a decrease in the relative number of fresh non-absorbing particles in the well mixed haze, while the total number of particles increases. No other changes are required and the rest of the atmospheric parameters remain stable.

In the case of scenario $\mathrm{B}$, instead, there is no need to change the average composition of the absorbing layer. The values of absorption are constant but the optical thickness of the "crème brûlée" layer changes by a factor of two, still at very low values so this is a 
subtle variation in the particle vertical distribution. This is accompanied by a much smaller change $(10 \%)$ in the optical thickness of the bottom cloud in the same region. It must be highlighted that the extension of the study to shorter wavelengths than those explored initially in the laboratory by Carlson et al. (2016) provides a hint of the width and central wavelength of the chromophore absorption band that merits future research.

Both scenarios, however, share in common a need to change the particle density at the upper troposphere levels in order to explain the changes in reflectivity witnessed after the disturbance of the North Temperate Belt. Stratospheric levels play no significant role and can thus be left aside for understanding this phenomenon. It is interesting to note that, even if both the particle number and absorption are equally responsible for the colouration, there is a $100 \%$ change in their number density in both scenarios.

We also want to mention that the discussion on color and altitude-opacity indices in section 3.2 were already pointing in the same direction as the detailed radiative transfer presented in section 4, showing an increase of the particle density in the upper troposphere together with a reddening of the average particle absorption. Although these indices are just a proxy to the real state of the atmosphere, they are based on a few filters and can be used for those cases where a more detailed analysis is not possible.

In fact, our retrievals are compatible with a change from scenario $\mathrm{A}$ to $\mathrm{B}$, or vice versa, during the disturbance of the NTB. As neither is clearly better than the other in reproducing the state of the atmosphere before or after the disturbance, it could be possible that the atmosphere changed from a situation in which the chromophore was isolated on top of the tropospheric haze (scenario B) to a situation closer to the well-mixed distribution in scenario A. This makes sense with the dynamical description of the disturbance, as turbulence and vertical motions might be able to increase the mixing of the chromophore within the upper tropospheric levels (Sánchez-Lavega et al., 2017). Conversely, the opposite is also compatible with the radiative transfer analysis, but might not be reconcilable with the observations. This mixed approach may require further dynamical analysis involving vertical and horizontal motions during and after the disturbance (Sánchez-Lavega et al., 2008).

The particles in the chromophore layer in scenario B seem to come from deeper levels, as the bottom layer suffers the opposite variation by a smaller amount within the parameter degeneracies discussed above, this provides a sense of vertical motion, together with an increase of the blue absorption. This is very interesting as this process is just the opposite in other coloration events of the opposite sign, as in the fade of the South Equatorial Belt (Pérez-Hoyos et al., 2012). So vertical motions from the ammonia cloud may play a role in the changes taking place closer to the tropopause, but this can result in redder or whiter clouds. Whether what comes from below is a pure non-absorbing species that reacts with other factors (such as solar UV radiation) or is originally red, remains unknown and cannot be discarded with the current data in hand.

With respect to the main chromophore absorption spectrum shown in Figure 10 we also retrieve in scenario A a steeper spectrum than the one proposed by Carlson et al. (2016). Our values are substantially lower than those in Braude et al. (2020) but this can be attributed to the vertical extension of the chromophore layer, which is much more concentrated in the latter and assumed to be composed by pure chromophore. While we provide a plausible extension of Carlson et al. (2016) imaginary refractive index to shorter wavelengths, scenario A favors quite a different spectral slope, which deserves further investigation.

Finally, regarding to the question of whether there is a single universal chromophore or two or more absorbing species (Simon-Miller et al., 2001b; Ordonez-Etxeberria et al., 2016), this work alone cannot provide any conclusive answer. For the current level of accuracy fitting the observed reflectivity, we can explain the spectra of these regions and epochs using a single chromophore species, irrespective of whether we use an extended 
or concentrated chromophore. Without analyzing more latitudes and epochs, we cannot support that this might be an universal chromophore, as this requires further research with an extended dataset far beyond the scope of this paper, but the similarity with the spectral slope retrieved by (Braude et al., 2020) is noteworthy. Here, the combination of imaginary refractive index and particle size is able to fit the observed spectral slope in 2016 and 2017 in the latitude range between $15^{\circ} \mathrm{N}$ and $40^{\circ} \mathrm{N}$.

\section{Conclusions}

In this work, we have analyzed Hubble Space Telescope images taken with the WFC3 camera in filters ranging from the near UV to the near IR in order to determine the most likely distribution of particles in the lower stratosphere and upper troposphere able to match the latitudinal and temporal variations of the reflectivity, including the observed limb-darkening. In order to do so, we have used two competing scenarios: one with an extended chromophore (A), and one with the chromophore concentrated on top of the tropospheric haze (B). The main conclusions of this work can be summarized as follows:

- Observed changes affect the nadir-viewing reflectivity and, less importantly, the limbdarkening coefficient.

- Changes are greatest (up to 60\%) at shorter wavelengths and in the deep methane band filter at $889 \mathrm{~nm}$.

- Changes are strongly concentrated in the region between $20^{\circ} \mathrm{N}$ and $25^{\circ} \mathrm{N}$, although they can be detected in a more extended region.

- This suggests a meridional propagation of the NTB activity outside the jet latitudes, but this has not been dynamically characterized and is outside the scope of this paper.

- In terms of the altitude/opacity and color indices, these regions show an increase after the NTBD in the altitude/opacity of the aerosols and in their blue absorption.

- Scenario A and B are both able to reproduce the observed reflectivity before and after the NTB disturbance, as well as its variation with wavelength and illumination/observation geometry.

- However, scenario A provides overall better fits, with the exception of limb-darkening at FQ889N in 2017, which is done better by scenario B.

- Scenario A requires an increase of almost a factor of 2 in the concentration of tropospheric haze particles, together with a stronger absorption at blue wavelengths.

- Scenario B requires an increase of $100 \%$ in the density of the chromophore layer, which is accompanied by a decrease of the particle number density at lower levels.

- For the first time, we extend the particle spectrum in scenario B below $400 \mathrm{~nm}$ and find a possible maximum absorption at $350 \mathrm{~nm}$.

- Both scenarios imply an upward displacement of particle, possibly from levels around the ammonia condensation levels to the upper troposphere.

- Limb-darkening fitting also favours a steeper slope of the chromophore absorption than that proposed by Carlson et al. (2016).

- The spectral slope of the chromophore absorption is similar to that proposed by Braude et al. (2020) as a possible universal chromophore. However, our more extended chromophore layer results in much lower absolute values of the effective imaginary refractive index, as we do not have a pure chromophore layer.

This work, together with that by Braude et al. (2020), is just one of the first works that tries to investigate the fit of the so-called "créme-brûlee" model to the observed limbdarkening at visible and neighbouring wavelengths. Although our main conclusion is that this is still better reproduced by an extended chromophore layer, there is still more work to be done in the near future to increase our knowledge of the Jovian chromophore and its nature. For this question to be answered models simultaneously fitting the geometrical and spectral reflectivity of the atmosphere is required, but also other aspects are mandatory. 
This is the case of laboratory experiments as the ones by Carlson et al. (2016), or detailed photochemical modelling of the atmosphere that validates the abundance of the required compounds at the expected levels.

\section{Acknowledgments}

This work was supported by the Spanish MICIIN projects AYA2015-65041-P (MINECO/FEDER, UE) and Grupos Gobierno Vasco IT-765-13 and IT1366-19, and UFI11/55 from UPV/EHU. This work used data acquired from the NASA/ESA HST Space Telescope, associated with OPAL program (PI: Simon, GO13937), and archived by the Space Telescope Science Institute, which is operated by the Association of Universities for Research in Astronomy, Inc., under NASA contract NAS 5-26555. All maps are available at http://dx.doi.org/10.17909/T9G593.

\section{References}

Astronomical Almanac. (1994). The Astronomical Almanac for the year 1995. Data for astronomy, space sciences, geodesy, surveying, navigation and other applications.

Baines, K. H., Sromovsky, L. A., Carlson, R. W., Momary, T. W., \& Fry, P. M. (2019, Sep). The visual spectrum of Jupiter's Great Red Spot accurately modeled with aerosols produced by photolyzed ammonia reacting with acetylene. Icarus, 330, 217-229. doi: 10.1016/j.icarus.2019.04.008

Barrado-Izagirre, N., Pérez-Hoyos, S., \& Sánchez-Lavega, A. (2009, Jul). Brightness power spectral distribution and waves in Jupiter's upper cloud and hazes. Icarus, 202(1), 181-196. doi: 10.1016/j.icarus.2009.02.015

Braude, A. S. (2019). Colour and cloud structure in the atmospheres of the giant planets (Unpublished doctoral dissertation). University of Oxford.

Braude, A. S., Irwin, P., Orton, G., \& Fletcher, L. (2020, Dec). Colour and Tropospheric Cloud Structure of Jupiter from MUSE/VLT: Retrieving a Universal Chromophore. Icarus, 338. doi: 10.1016/j.icarus.2019.113589

Carlson, R. W., Baines, K. H., Anderson, M. S., Filacchione, G., \& Simon, A. A. (2016, Aug). Chromophores from photolyzed ammonia reacting with acetylene: Application to Jupiter's Great Red Spot. Icarus, 274, 106-115. doi: 10.1016/j.icarus.2016.03.008

Coles, P. A., Ovsyannikov, R. I., Polyansky, O. L., Yurchenko, S. N., \& Tennyson, J. (2018, November). Improved potential energy surface and spectral assignments for ammonia in the near-infrared region. Journal of Quant. Spec. and Rad. Trans., 219, 199-212. doi: $10.1016 /$ j.jqsrt.2018.07.022

de Pater, I., Fletcher, L. N., Pérez-Hoyos, S., Hammel, H. B., Orton, G. S., Wong, M. H., ... Boslough, M. (2010, December). A multi-wavelength study of the 2009 impact on Jupiter: Comparison of high resolution images from Gemini, Keck and HST. Icarus, 210(2), 722-741. doi: 10.1016/j.icarus.2010.07.010

de Pater, I., Wong, M. H., Marcus, P., Luszcz-Cook, S., Ádámkovics, M., Conrad, A., .. Go, C. (2010, Dec). Persistent rings in and around Jupiter's anticyclones - Observations and theory. Icarus, 210(2), 742-762. doi: 10.1016/j.icarus.2010.07.027

Dressel, L. (2019). Wide Field Camera 3 Instrument Handbook, Version 11.0.

García-Melendo, E., Sánchez-Lavega, A., \& Dowling, T. E. (2005, Aug). Jupiter's 24N highest speed jet: Vertical structure deduced from nonlinear simulations of a largeamplitude natural disturbance. Icarus, 176 (2), 272-282. doi: 10.1016/j.icarus.2005.02 .012

Hanel, R. A., Conrath, B. J., Jennings, D. E., \& Samuelson, R. E. (1992). Exploration of the solar system by infrared remote sensing.

Horak, H. G. (1950, Nov). Diffuse Reflection by Planetary Atmospheres. Astrophys. Journal, 112, 445. doi: 10.1086/145359

Hueso, R., Legarreta, J., García-Melendo, E., Sánchez-Lavega, A., \& Pérez-Hoyos, S. (2009, Oct). The jovian anticyclone BA. II. Circulation and interaction with the zonal jets. Icarus, 203(2), 499-515. doi: 10.1016/j.icarus.2009.05.004 
Irwin, P. G. J., Teanby, N. A., de Kok, R., Fletcher, L. N., Howett, C. J. A., Tsang, C. C. C., ... Parrish, P. D. (2008, April). The NEMESIS planetary atmosphere radiative transfer and retrieval tool. Journal of Quant. Spec. and Radiative Transfer, 109, 1136-1150. doi: 10.1016/j.jqsrt.2007.11.006

Karkoschka, E. (1994, September). Spectrophotometry of the Jovian Planets and Titan at 300- to 1000-nm Wavelength: The Methane Spectrum. Icarus, 111(1), 174-192. doi: 10.1006/icar.1994.1139

Karkoschka, E. (1998, May). Methane, Ammonia, and Temperature Measurements of the Jovian Planets and Titan from CCD-Spectrophotometry. Icarus, 133(1), 134-146. doi: $10.1006 /$ icar.1998.5913

Karkoschka, E., \& Tomasko, M. G. (2010, February). Methane absorption coefficients for the jovian planets from laboratory, Huygens, and HST data. Icarus, 205(2), 674-694. doi: 10.1016/j.icarus.2009.07.044

Martonchik, J. V., Orton, G. S., \& Appleby, J. F. (1984, Feb). Optical properties of $\mathrm{NH}_{3}$ ice from the far infrared to the near ultraviolet. Applied Optics, 23, 541-547. doi: 10.1364/AO.23.000541

Minnaert, M. (1941, May). The reciprocity principle in lunar photometry. Astrophys. Journal, 93, 403-410. doi: 10.1086/144279

Ordonez-Etxeberria, I., Hueso, R., Sánchez-Lavega, A., \& Pérez-Hoyos, S. (2016, Mar). Spatial distribution of jovian clouds, hazes and colors from Cassini ISS multi-spectral images. Icarus, 267, 34-50. doi: 10.1016/j.icarus.2015.12.008

Owen, T., \& Terrile, R. J. (1981, Sep). Colors on Jupiter. Journal of Geophys. Res. (Planets), 86(A10), 8797-8814. doi: 10.1029/JA086iA10p08797

Pérez-Hoyos, S., Sánchez-Lavega, A., Hueso, R., García-Melendo, E., \& Legarreta, J. (2009, Oct). The jovian anticyclone BA. III. Aerosol properties and color change. Icarus, 203(2), 516-530. doi: 10.1016/j.icarus.2009.06.024

Pérez-Hoyos, S., Sanz-Requena, J. F., Barrado-Izagirre, N., Rojas, J. F., Sánchez-Lavega, A., \& IOPW Team. (2012, Jan). The 2009-2010 fade of Jupiter's South Equatorial Belt: Vertical cloud structure models and zonal winds from visible imaging. Icarus, 217(1), 256-271. doi: 10.1016/j.icarus.2011.11.008

Pérez-Hoyos, S., Sanz-Requena, J. F., Sánchez-Lavega, A., Irwin, P. G. J., \& Smith, A. (2016, Oct). Saturn's tropospheric particles phase function and spatial distribution from Cassini ISS 2010-11 observations. Icarus, 277, 1-18. doi: 10.1016/j.icarus.2016 .04 .022

Rodgers, C. D. (2000). Inverse Methods for Atmospheric Sounding: Theory and Practice. doi: $10.1142 / 3171$

Rogers, J. H. (2009). The Giant Planet Jupiter. Cambridge University Press, Cambridge, UK.

Rogers, J. H., \& Adamoli, G. (2019, June). Jupiter's North Equatorial Belt \&amp; Jet Part III: The 'great northern upheaval' in 2012. Journal of the British Astronomical Association, 129, 158-169.

Sánchez-Lavega, A., Legarreta, J., García-Melendo, E., Hueso, R., Pérez-Hoyos, S., GómezForrellad, J. M., ... Cecconi, M. (2013, Dec). Colors of Jupiter's large anticyclones and the interaction of a Tropical Red Oval with the Great Red Spot in 2008. Journal of Geophys. Res. (Planets), 118(12), 2537-2557. doi: 10.1002/2013JE004371

Sánchez-Lavega, A., Orton, G. S., Hueso, R., García-Melendo, E., Pérez-Hoyos, S., SimonMiller, A., .. Pujic, Z. (2008, Feb). Depth of a strong jovian jet from a planetary-scale disturbance driven by storms. Nature, 451 (7181), 1022. doi: 10.1038/nature06807

Sánchez-Lavega, A., Rogers, J. H., Orton, G. S., García-Melendo, E., Legarreta, J., Colas, F., ... Wesley, A. (2017, May). A planetary-scale disturbance in the most intense Jovian atmospheric jet from JunoCam and ground-based observations. Geophys. Res. Lett., 44, 4679-4686. doi: 10.1002/2017GL073421

Sanz-Requena, J. F., Pérez-Hoyos, S., Sánchez-Lavega, A., Antuñano, A., \& Irwin, P. G. J. (2018, May). Haze and cloud structure of Saturn's North Pole and Hexagon Wave from Cassini/ISS imaging. Icarus, 305, 284-300. doi: 10.1016/j.icarus.2017.12.043 
Sanz-Requena, J. F., Pérez-Hoyos, S., Sánchez-Lavega, A., del Rio-Gaztelurrutia, T., \& Irwin, P. G. J. (2019, Nov). Hazes and clouds in a singular triple vortex in Saturn's atmosphere from HST/WFC3 multispectral imaging. Icarus, 333, 22-36. doi: 10.1016/ j.icarus.2019.05.037

Seiff, A., Kirk, D., Knight, T., Young, R., Mihalov, J., Young, L., ... Atkinson, D. (1998). Thermal structure of jupiter's atmosphere near the edge of a 5 - $\mu \mathrm{m}$ hot spot in the north equatorial belt. Journal of Geophysical Research E: Planets, 103(E10), 22857-22889. Retrieved from https : //www . scopus. com/inward/record . uri?eid=2-s2.0-0032566866\&doi= 10. $1029 \% 2$ f98JE01766\&partner ID $=40 \& \mathrm{md} 5=140 \mathrm{f} 03 \mathrm{c} 80 \mathrm{e} 1 \mathrm{~b} 4675918282 \mathrm{e} 6 \mathrm{f} \mathrm{cc} 80 \mathrm{~d} 50$ (cited By 193) doi: 10.1029/98JE01766

Simon, A. A., Wong, M. H., \& Orton, G. S. (2015, Oct). First Results from the Hubble OPAL Program: Jupiter in 2015. Astrophys. Journal, 812(1), 55. doi: 10.1088/ 0004-637X/812/1/55

Simon-Miller, A. A., Banfield, D., \& Gierasch, P. J. (2001a, Dec). Color and the Vertical Structure in Jupiter's Belts, Zones, and Weather Systems. Icarus, 154(2), 459-474. doi: 10.1006/icar.2001.6742

Simon-Miller, A. A., Banfield, D., \& Gierasch, P. J. (2001b, Jan). An HST Study of Jovian Chromophores. Icarus, $149(1)$, 94-106. doi: 10.1006/icar.2001.6473

Sromovsky, L. A., Baines, K. H., Fry, P. M., \& Carlson, R. W. (2017, Jul). A possibly universal red chromophore for modeling color variations on Jupiter. Icarus, 291, 232244. doi: $10.1016 /$ j.icarus.2016.12.014

Strycker, P. D., Chanover, N. J., Simon-Miller, A. A., Banfield, D., \& Gierasch, P. J. (2011, Oct). Jovian chromophore characteristics from multispectral HST images. Icarus, 215(2), 552-583. doi: 10.1016/j.icarus.2011.08.004

Taylor, F. W., Atreya, S. K., Encrenaz, T., Hunten, D. M., Irwin, P. G. J., \& Owen, T. C. (2004). The composition of the atmosphere of Jupiter. In F. Bagenal, T. E. Dowling, \& W. B. McKinnon (Eds.), Jupiter. the planet, satellites and magnetosphere (Vol. 1, p. 59-78).

West, R. A., Baines, K. H., Friedson, A. J., Banfield, D., Ragent, B., \& Taylor, F. W. (2004). Jovian clouds and haze. In F. Bagenal, T. E. Dowling, \& W. B. McKinnon (Eds.), Jupiter. the planet, satellites and magnetosphere (Vol. 1, p. 79-104).

Zhang, X., West, R. A., Banfield, D., \& Yung, Y. L. (2013, September). Stratospheric aerosols on Jupiter from Cassini observations. Icarus, 226(1), 159-171. doi: 10.1016/ j.icarus.2013.05.020 